\title{
Envelopes, indicators and conservativeness
}

\author{
Andrés Cordón-Franco, Alejandro Fernández-Margarit, and F.Félix Lara-Martín \\ Departamento de Ciencias de la Computación e Inteligencia Artificial, Facultad de Matemáticas, Universidad de Sevilla, C/ \\ Tarfia s/n, 41012 Sevilla, Spain
}

Key words Collection principles, initial segments, indicators, envelopes, conservation results.

well known theorem proved (independently) by J. Paris and H. Friedman states that $\mathbf{B} \Sigma_{n+1}$ (the fragment of Arithmetic given by the collection scheme restricted to $\Sigma_{n+1}$-formulas) is a $\Pi_{n+2}$-conservative extension of $\mathbf{I} \Sigma_{n}$ (the fragment given by the induction scheme restricted to $\Sigma_{n}$-formulas). In this paper, as a continuation of our previous work on collection schemes for $\Delta_{n+1}(T)$-formulas (see [4]), we study a general version of this theorem and characterize theories $T$ such that $T+\mathbf{B} \Sigma_{n+1}$ is a $\Pi_{n+2}$-conservative extension of $T$. We prove that this conservativeness property is equivalent to a model-theoretic property relating $\Pi_{n}$-envelopes and $\Pi_{n}$-indicators for $T$. The analysis of $\Sigma_{n+1}$-collection we develop here is also applied to $\Sigma_{n+1^{-}}$ induction using Parsons' conservativeness theorem instead of Friedman-Paris' theorem.

As a corollary, our work provides new model-theoretic proofs of two theorems of R. Kaye, J. Paris and C. Dimitracopoulos (see [8]): $\mathbf{B} \Sigma_{n+1}$ and $\mathbf{I} \Sigma_{n+1}$ are $\Sigma_{n+3}$-conservative extensions of their parameter free versions, $\mathbf{B} \Sigma^{-} \cdot{ }_{n+1 n+1}$ and $\mathbf{I} \Sigma^{-}$

\section{Introduction}

In studying the class of provably total functions of a fragment of Arithmetic a useful idea is to isolate a subclass of those functions with good growth properties. Typically, one obtains a sequence of increasing functions of ever-faster rate of growth such that each provably total function is bounded by some function in the sequence. This kind of constructions not only provides descriptions of the class of provably total functions but also of the class of the $\Pi_{2}$-consequences of the considered fragment. In this way, they also constitute a useful tool to obtain $\Pi_{2}$-conservation results.

A basic principle involved in those arguments is the collection principle. In one of its formulations this principle asserts that every total function can be bounded by an increasing one. Hence, there exist natural relations between conservation properties for collection principles and conditions under which the above mentioned constructions can be carried out. In a model-theoretic setting previous ideas occur notably in the theory of indicators and envelopes of fragments of Arithmetic but, in some aspects, only in an implicit and ad hoc form. In this paper we shall make explicit this relationship and develop a general theory on this topic.

Indicators were introduced by L. Kirby and J. Paris in [9] (see also [12]) as a tool to study the distribution in models of Peano Arithmetic of initial segments satisfying a given property. Paris and Kirby (see [14] or [10]) developed the theory of indicators in countable models of Peano Arithmetic. However, as noticed, for example, in [11], it is possible to develop a general theory of indicators in countable models of theories weaker than Peano Arithmetic. Indeed, in [15] Paris stated the main results on indicators for theories extending $\mathbf{I} \Sigma_{1}$, see [15, Theorem 0]. Here, we shall consider a more concrete (but still general enough) notion of indicator in (countable) models of theories that can prove a weak form of collection. In [4], these theories have been called $\Pi_{n}$-functional. 
On the other hand, the notion of an envelope was first isolated by K. McAloon in [13]. There envelopes were used to give an alternative treatment to some results previously formulated in terms of indicators. However, envelopes were implicit in Paris' first works on indicators (see [14]) as a tool to characterize the class of provably total recursive functions of a theory.

Since that early work it is well known that it is possible to obtain an envelope from an indicator. A general version of this fact, for indicators for theories extending $\mathbf{I} \Sigma_{1}$, is proved in P. Hájek and P. Pudlák's book [5]. Conversely, in certain cases, it is possible to get an indicator from an envelope. This suggests that in some sense envelopes and indicators are equivalent notions. The principal aim of this work is to provide a precise formulation of this equivalence by explicitly stating the conditions under which it holds.

In order to get those conditions a general theory of envelopes is needed. Such a general theory was developed in [4] elaborating on the material on indicators included by R. Kaye in his book [7]. There he introduced the concept of a $\Pi_{n}$-envelope of a theory $T$ in another theory $T_{0}$. As it is apparent from our work in [4], $\Pi_{n}$-functional theories are the proper context to deal with $\Pi_{n}$-envelopes. Roughly speaking a theory is $\Pi_{n}$-functional if its $\Pi_{n+2}$-consequences can be described by $\mathbf{I} \Sigma_{n}$ plus a class of nondecreasing $\Pi_{n}$-functions. In this case, (if there exists) a $\Pi_{n}$-envelope provides a uniform description of such a family of nondecreasing functions. Here we study the equivalence between $\Pi_{n}$-envelopes and $\Pi_{n}$-indicators in this context. The key property to obtain that equivalence is a general version of Friedman-Paris' $\Pi_{n+2}$-conservativeness theorem between $\mathbf{B} \Sigma_{n+1}$ and $\mathbf{I} \Sigma_{n}$. That property was considered in [4], where we asked the following question (see [4, Problem 7.3]):

(P) Let $T$ be a theory such that $T+\mathbf{B} \Sigma_{n+1}$ is consistent. Are the following conditions equivalent?

(a) $T$ is a $\Pi_{n}$-functional theory.

(b) $T+\mathbf{B} \Sigma_{n+1}$ is $\Pi_{n+2}$-conservative over $T$.

Since property (b) will play a central role through this paper, we introduce the following definition.

Definition 1.1 We say that a theory $T$ is $\Pi_{n+2}^{\mathbf{B}}$-conservative if $T+\mathbf{B} \Sigma_{n+1}$ is $\Pi_{n+2}$-conservative over $T$; that is, $\operatorname{Th}_{\Pi_{n+2}}\left(T+\mathbf{B} \Sigma_{n+1}\right)=\operatorname{Th}_{\Pi_{n+2}}(T)$.

Using this definition Friedman-Paris' theorem can be stated as: $\mathbf{I} \Sigma_{n}$ is $\Pi_{n+2}^{\mathbf{B}}$-conservative. L. Beklemishev showed in [2, Theorem 3.2] that if $T$ is a $\Pi_{n+3}$-axiomatizable theory (proving that exponential function is total) and it is closed under $\Sigma_{n+1}$-collection rule, then it is $\Pi_{n+2}^{\mathbf{B}}$-conservative. (Collection rule is defined by

$\mathrm{CR}: \quad \frac{\forall x \exists y \varphi(x, y)}{\forall z \exists u(\forall x \leq z)(\exists y \leq u) \varphi(x, y)}$.

For a theory $T, T+\Sigma_{n+1}$-CR denotes the closure of $T$ under first-order logic and applications of CR restricted to formulas $\varphi \in \Sigma_{n+1}$.)

It is easy to see that every $\Pi_{n+2}^{\mathrm{B}}$-conservative theory is closed under $\Sigma_{n+1}$-collection rule. Therefore, $\Pi_{n+2}^{\mathrm{B}}$-conservativeness is equivalent to the closure under $\Sigma_{n+1}$-collection rule, for $\Pi_{n+3}$-axiomatizable theories. This fact is used in [2] to derive a proof-theoretic proof of Friedman-Paris' theorem. In addition, it also provides a partial answer to problem $(\mathbf{P})$, as long as the theories closed under $\Sigma_{n+1}$-collection rule are exactly the $\Pi_{n}$-functional ones (see Theorems 1.3 and 1.4 below).

In this paper we address a general answer to problem $(\mathbf{P})$ and provide a model-theoretic analysis of this question. Our work relates $\Pi_{n+2}^{\mathrm{B}}$-conservativeness to a model-theoretic property of $\Pi_{n}$-envelopes. Roughly speaking, that property (that we call $\Pi_{n}$-IND) asserts that $\Pi_{n}$-envelopes define $\left(\Pi_{n}\right.$-)indicators, for precise definitions see Section 2. The analysis of $\Pi_{n}$-IND was initiated in [4] in connection with our work on the collection scheme for $\Delta_{n+1}(T)$-formulas. Here, we continue that analysis pointing out a close relation between conservation results on $\Sigma_{n+1}$-collection (including both axioms and rules) and natural conditions to develop a general theory of indicators and envelopes.

In order to present more precisely the results obtained in this paper and their connection with the above conservation property we state some general notation and recall some concepts and results from [4]. We shall work in the first-order language of Arithmetic, $\mathcal{L}$. As usual, we denote by exp a $\Pi_{2}$-sentence expressing that exponential function is total. As above, $T$ will denote a consistent theory in the language $\mathcal{L}$. For such a theory we introduced the classes of formulas

$$
\Delta_{n+1}(T)=\left\{\varphi(x) \in \Sigma_{n+1}: \text { there exists } \psi(x) \in \Pi_{n+1}, T \vdash \varphi \leftrightarrow \psi\right\} .
$$


When the schemes of induction and minimization are restricted to these classes of formulas we obtain the theories $\mathbf{I} \Delta_{n+1}(T)$ and $\mathbf{L} \Delta_{n+1}(T)$. We also consider the following version of the collection scheme:

$$
\mathbf{B}^{*} \Delta_{n+1}(T)=\mathbf{I} \Delta_{0}+\left\{B_{\varphi, x, y}: \varphi \in \Pi_{n}, \exists y \varphi(x, y) \in \Delta_{n+1}(T)\right\},
$$

where $B_{\varphi, x, y}(z, \vec{v})$ denotes the collection axiom for $\varphi(x, y, \vec{v})$ with respect to $x, y$; that is, the formula

$$
(\forall x \leq z) \exists y \varphi(x, y, \vec{v}) \rightarrow \exists u(\forall x \leq z)(\exists y \leq u) \varphi(x, y, \vec{v}) .
$$

In [4] and [3] properties and relative strength of these schemes were studied. We introduced, among others, the following notion:

Definition 1.2 We say that a theory $T$ has $\Delta_{n+1}$-collection if $T \Rightarrow \mathbf{B}^{*} \Delta_{n+1}(T)$.

The main feature of theories having $\Delta_{n+1}$-collection is that for every such a theory $T$, the class of $\Delta_{n+1}(T)$-formulas is closed in $T$ under bounded quantification. From this basic fact we derived in [4] that if $T$ has $\Delta_{n+1}$-collection, then $T$ extends $\mathbf{L} \Delta_{n+1}(T)$ and $\mathbf{I} \Delta_{n+1}(T)$.

It is not difficult to prove (see [4, Remark 2.8]) that an extension of $\mathbf{I} \Delta_{0}$ has $\Delta_{n+1}$-collection if and only if it is closed under $\Sigma_{n+1}$-collection rule. Besides this proof-theoretic characterization of $\Delta_{n+1}$-collection we obtained a "functional" characterization more amenable to model-theoretic investigation. This is precisely the notion of a $\Pi_{n}$-functional theory. We need some notation to state it.

In what follows, $\Gamma$ will denote a class of formulas of $\mathcal{L}$ with two free variables, $x, y$ say. For a formula $\varphi(x, y)$, $\operatorname{IPF}(\varphi)$ will denote a formula expressing that $\varphi(x, y)$ defines a nondecreasing (partial) function. Let

$$
\Gamma^{*}=\{\operatorname{IPF}(\varphi(x, y)): \varphi(x, y) \in \Gamma\} \cup\{\forall x \exists ! y \varphi(x, y): \varphi(x, y) \in \Gamma\} .
$$

Let us observe that if $\Gamma \subseteq \Pi_{n}$, then $\Gamma^{*}$ is a set of $\Pi_{n+2}$-sentences expressing that each formula in $\Gamma$ defines a nondecreasing total function. We consider the following concepts:

1. We say that $\Gamma \subseteq \Pi_{n}$ is a $\Pi_{n}$-functional class if $\mathbf{I} \Sigma_{n}+\Gamma^{*}$ is consistent.

2. A theory $T$ is $\Pi_{n}$-functional if there exists a $\Pi_{n}$-functional class $\Gamma$ such that

$$
\operatorname{Th}_{\Pi_{n+2}}(T)=\operatorname{Th}_{\Pi_{n+2}}\left(\mathbf{I} \Sigma_{n}+\Gamma^{*}\right) .
$$

The class of formulas $\Delta_{n+1}(T)$ is determined by the $\Pi_{n+2}$-consequences of $T$, so it is natural to look for a characterization of the theories having $\Delta_{n+1}$-collection which relies on the description of $\operatorname{Th}_{\Pi_{n+2}}(T)$. This is achieved through the notion of a $\Pi_{n}$-functional theory. The next theorem sums up the equivalence among the three notions we have introduced (see [4, Theorem 3.5 and Remark 2.8]). For $n=0$ a similar result was obtained by L. Beklemishev in [1, Proposition 5.4].

Theorem 1.3 Let $T$ be a consistent theory. The following conditions are equivalent:

1. $T$ has $\Delta_{n+1}$-collection.

2. $T$ is $\Pi_{n}$-functional.

3. $T$ is an extension of $\mathbf{I} \Delta_{0}$ closed under $\Sigma_{n+1}$-collection rule; that is, $T \Leftrightarrow T+\Sigma_{n+1}$-CR.

In view of this result and our previous remarks on Beklemishev's work ([2, Theorem 3.2]) we can state the following theorem.

Theorem 1.4 Let $T$ be a $\Pi_{n+3}$-axiomatizable and consistent theory. The following conditions are equivalent:

(1) $T$ is $\Pi_{n}$-functional.

(2) $T$ is $\Pi_{n+2}^{\mathrm{B}}$-conservative.

In this paper we present a model-theoretic proof of this result. In [4, Remark 3.7.2], we proved this theorem for $\Pi_{n+2}$-axiomatizable theories. Here, we give an alternative proof for the $\Pi_{n+2}$ case and we extend it to $\Pi_{n+3}$-axiomatizable theories as an application of our work on $\Pi_{n}$-envelopes. Since $T+\mathbf{B} \Sigma_{n+1}$ has $\Delta_{n+1}$-collection, part (2) $\Rightarrow(1)$ easily follows from Theorem 1.3. Hence, our concern here will be the converse. Moreover, we will show that Theorem 1.4 is optimal with respect to the syntactical complexity of the theory (see Remark 3.10 ) and this answers problem $(\mathbf{P})$.

The paper is organized as follows. In Section 2 we introduce the basic notions we will deal with in this paper: $\Pi_{n}$-envelopes, $\Pi_{n}$-indicators and the property $\Pi_{n}$-IND. We describe the relationship between property $\Pi_{n}$-IND and $\Pi_{n+2}^{\mathrm{B}}$-conservativeness and obtain the results on $\Pi_{n}$-envelopes that constitute the real core of this paper. 
Firstly, without restrictions on the syntactical complexity of the theory, we characterize recursively axiomatizable and $\Pi_{n}$-functional theories (proving that exponential function is total) which are $\Pi_{n+2}^{\mathbf{B}}$-conservative. They are, namely, those theories whose $\Pi_{n}$-envelopes define (well-behaved) $\Pi_{n}$-indicators. The next theorem sums up the results obtained in Theorems 2.4 and 2.7 .

Theorem 1.5 Let $T$ be a recursively axiomatizable and $\Pi_{n}$-functional theory such that $T \vdash \exp$. The following conditions are equivalent:

1. $T$ is $\Pi_{n+2}^{\mathrm{B}}$-conservative.

2. There exists a $\Pi_{n}$-envelope of $T$ in $T$ satisfying $\Pi_{n}$-IND.

3. There exists a well-behaved $\Pi_{n}$-indicator, $Y(x, y)=z$, for $T$ in $T$ such that for all $k \in \omega$,

$$
T \vdash \forall x \exists y(Y(x, y)>k) .
$$

Secondly (see Theorem 2.10), we prove (a slightly sharper variation of) the following theorem.

Theorem 1.6 Let $T$ be a $\Pi_{n}$-functional theory such that $T \vdash \exp$. If $T$ is $\Pi_{n+3}$-axiomatizable, then every $\Pi_{n}$-envelope of $T$ in $T$ satisfies $\Pi_{n}$-IND.

From these results Theorem 1.4 can be derived for theories proving that exponential function is total (see Corollary 2.11). However, because known existence theorems for envelopes use the exponential function, the proof of Theorem 1.4 in its whole generality requires some additional work that will be done in Section 3 . To this end, the central result is Theorem 3.7 there. The proof of this theorem deals with the notion of a pseudo- $\Pi_{n}$-envelope and provides a model-theoretic tool for the analysis of collection rule. As we have noticed, the first condition of Theorem 1.4 is equivalent to the closure of $T$ under $\Sigma_{n+1}$-collection rule; so, the work presented here (and in [4]) can be considered as a model-theoretic analysis of collection rule (and, therefore, it is closely tied to Beklemishev's work in [2]). As a matter of fact, from Theorem 3.7 we derive [2, Theorem 3.2] (proved there by a cut-elimination argument) and a new model-theoretic proof of a conservativeness result between $\mathbf{B} \Sigma_{n+1}$ and $\mathbf{B} \Sigma_{n+1}^{-}$firstly proved by R. Kaye, J. Paris and C. Dimitracopoulos (see [8, Theorem 2.4]).

Finally, in Section 4 we apply the previously developed analysis of $\Sigma_{n+1}$-collection rule to $\Sigma_{n+1}$-induction. This can be done with minor changes. Now the basic result that plays the role of Friedman-Paris' theorem is Parsons' conservation theorem on $\Sigma_{n+1}$-induction rule. Induction rule is given by

IR : $\quad \frac{\varphi(0), \quad \forall x(\varphi(x) \rightarrow \varphi(x+1))}{\forall x \varphi(x)}$.

As for collection rule, $T+\Sigma_{n+1}$-IR denotes the closure of $T$ under first-order logic and applications of IR restricted to formulas $\varphi \in \Sigma_{n+1}$. C. Parsons proved that (see [16]):

Theorem 1.7 (Parsons) $\mathbf{I} \Sigma_{n+1}$ is a $\Pi_{n+2}$-conservative extension of $\mathbf{I} \Delta_{0}+\Sigma_{n+1}$-IR.

Since $\mathbf{I} \Sigma_{n}$ is equivalent to $\mathbf{I} \Delta_{0}+\Sigma_{n+1}-\mathrm{CR}$ (see [2, Corollary 4.2]), Friedman-Paris' theorem can be stated as: $\mathbf{B} \Sigma_{n+1}$ is a $\Pi_{n+2}$-conservative extension of $\mathbf{I} \Delta_{0}+\Sigma_{n+1}$-CR.

So, it is natural to introduce a notion of $\Pi_{n+2}^{\mathbf{I}}$-conservativeness and study it along the lines just described for the collection case.

Definition 1.8 A theory $T$ is $\Pi_{n+2}^{\mathbf{I}}$-conservative if $T+\mathbf{I} \Sigma_{n+1}$ is $\Pi_{n+2}$-conservative over $T$.

The main result on $\Sigma_{n+1}$-induction rule proved here is the following one:

Theorem 1.9 (see Theorem 4.1) Let $T$ be a $\Pi_{n+3}$-axiomatizable theory. Then we have:

$$
T \text { is closed under } \Sigma_{n+1} \text {-induction rule } \Leftrightarrow T \text { is } \Pi_{n+2}^{\mathbf{I}} \text {-conservative. }
$$

As for collection, Theorem 1.9 is optimal with respect to the syntactical complexity of the theory. It is easy to offer examples along the lines given in Remark 3.10 for the collection case. Moreover, Theorem 1.9 provides a new proof of [8, Theorem 2.1]: $\mathbf{I} \Sigma_{n+1}$ is a $\Sigma_{n+3}$-conservative extension of $\mathbf{I} \Sigma_{n+1}^{-}$.

The work presented in this paper shows the relationship between conservation (for $\mathbf{B} \Sigma_{n+1}$ and $\mathbf{B} \Sigma_{n+1}^{-}$) and consistency of theories of the form $T+\mathbf{B} \Sigma_{n+1}$, where $T$ is $\Pi_{n}$-functional. We conclude this section with a related question.

Problem 1.10 Let $\varphi \in \Pi_{n+2}$ be a sentence such that $\mathbf{L} \Delta_{n+1}^{-}+\varphi\left(\right.$ or $\left.\mathbf{L} \Delta_{n+1}^{-}+\varphi+\mathbf{I} \Sigma_{n}\right)$ is a consistent theory. Is there a $\Pi_{n}$-functional theory $T$ such that $T \vdash \varphi$ ?

If yes, then $\mathbf{B} \Sigma_{n+1}$ is $\Sigma_{n+2}$-conservative over $\mathbf{L} \Delta_{n+1}^{-}$(or over $\mathbf{L} \Delta_{n+1}^{-}+\mathbf{I} \Sigma_{n}$ ). Let us prove it. 
Let $\varphi \in \Sigma_{n+2}$ be a sentence such that $\mathbf{B} \Sigma_{n+1} \vdash \varphi$. If $\mathbf{L} \Delta_{n+1}^{-}\left(+\mathbf{I} \Sigma_{n}\right) \forall \varphi$, then $\mathbf{L} \Delta_{n+1}^{-}+\neg \varphi\left(+\mathbf{I} \Sigma_{n}\right)$ is consistent. By hypothesis, there exists a $\Pi_{n}$-functional theory $T$ such that $T \vdash \neg \varphi$. Since $\neg \varphi$ is $\Pi_{n+2}$, we may assume that $T$ is $\Pi_{n+2}$-axiomatizable. Hence, by Theorem 1.4, $T+\mathbf{B} \Sigma_{n+1}$ is consistent. Since $T \vdash \neg \varphi$ and $\mathbf{B} \Sigma_{n+1} \vdash \varphi$, this gives the desired contradiction.

\section{$2 \Pi_{n}$-envelopes and $\Pi_{n}$-indicators}

In what follows we introduce the basic notions we will deal with in this paper: $\Pi_{n}$-envelopes, $\Pi_{n}$-indicators and the property $\Pi_{n}$-IND. We describe the relationship between property $\Pi_{n}$-IND and $\Pi_{n+2}^{\mathrm{B}}$-conservativeness and obtain the main results relating $\Pi_{n}$-envelopes and $\Pi_{n}$-indicators.

\subsection{The property $\Pi_{n}$-IND}

In this section we give a short review of some relevant facts of the theory of $\Pi_{n}$-envelopes as developed in [4]. In this way, in addition to present the exact definitions of the notions involved we will make explicit the connection between $\Pi_{n}$-envelopes and $\Pi_{n+2}^{\mathrm{B}}$-conservativeness.

To get a uniform description of $\Pi_{n}$-functional classes, in [4] we introduced the concept of a $\Pi_{n}$-envelope.

Definition 2.1 Let $T, T_{0}$ be consistent theories, $\varphi(u, x, y) \in \Sigma_{n+1}$ and $\Gamma_{\varphi}=\{\varphi(k, x, y): k \in \omega\}$. We say:

(1) $\varphi(u, x, y)$ is a $\Pi_{n}$-q-envelope of $T$ in $T_{0}$ if

(a) $T \vdash \Gamma_{\varphi}^{*}$; that is, for all $k \in \omega, T \vdash \operatorname{IPF}(\varphi(k, x, y)) \wedge \forall x \exists y \varphi(k, x, y)$;

(b) for all $k \in \omega, T_{0} \vdash \varphi(k+1, x, y) \rightarrow(\exists z<y) \varphi(k, x, z)$.

(2) $\varphi(u, x, y)$ satisfies $\Pi_{n}$-ENV for $T$ and $T_{0}$ if for each $\psi(x, y) \in \Pi_{n}$ such that $T \vdash \forall x \exists y \psi(x, y)$, there exists $k \in \omega$ such that $T_{0} \vdash \varphi(k, x, y) \rightarrow(\exists z<y) \psi(x, z)$. (By a standard quantifier contraction argument this property also holds for $\psi(x, y) \in \Sigma_{n+1}$.)

(3) $\varphi(u, x, y)$ is a $\Pi_{n}$-envelope of $T$ in $T_{0}$ if $\varphi(u, x, y)$ is a $\Pi_{n}$-q-envelope of $T$ in $T_{0}$ and satisfies $\Pi_{n}$-ENV for $T$ and $T_{0}$.

This definition describes a very general notion and, at a first sight, the role of $T_{0}$ could be seen as artificial; so, some comments are in order. Actually, in most of the cases, it can be assumed that $T_{0}$ is $\mathbf{I} \Sigma_{n}+\exp$. This assumption would simplify the statements of the results in this section; and the reader can assume that this is the case until Section 3 . However, when dealing with the weaker notion of a pseudo- $\Pi_{n}$-envelope in Section 3 , it will be useful to consider different choices of $T_{0}$. So, we prefer to make explicit the role of $T_{0}$ from the very beginning.

Let us denote the formula $\varphi(u, x, y)$ by $F_{u}(x)=y$. Then, roughly speaking, a $\Pi_{n}$-envelope is a $\Sigma_{n+1}$-definable sequence of provably total nondecreasing functions, $\left\{F_{k}\right\}_{k \in \omega}$. Its essential feature is property $\Pi_{n}-\mathrm{ENV}$ expressed in item (2): Every $\Pi_{n}$-definable (or even $\Sigma_{n+1}$-definable) function provably total in $T$ is (provably in $T_{0}$ ) majorized by a function in the sequence. The remaining properties stated in (1) are of technical character. Sequences satisfying these properties have been called $\Pi_{n}$-q-envelopes in order to discuss alternatives to $\Pi_{n}$-ENV. The following property can be considered as a model-theoretic strong version of $\Pi_{n}-E N V$. It captures a common property of envelopes defined from indicators.

Definition 2.2 Let $\varphi(u, x, y) \in \Sigma_{n+1}$. We say $\varphi(u, x, y)$ satisfies $\Pi_{n}$-IND for $T$ and $T_{0}$ if for every $\mathfrak{A} \vDash T_{0}$ countable, nonstandard and $a, b \in \mathfrak{A}$, the following conditions are equivalent:

IND-(i): For all $k \in \omega, \mathfrak{A} \vDash(\exists y<b) \varphi(k, a, y)$.

IND-(ii): There exists $I \vDash T$ such that $I \prec_{n}^{\mathrm{e}} \mathfrak{A}$ and $a<I<b$.

In [4] we obtained conditions under which $\Pi_{n}$-ENV is equivalent to $\Pi_{n}$-IND. As we noticed there, if $T_{0}$ extends $\mathbf{I} \Sigma_{n}$, then every $\Pi_{n}$-q-envelope of $T$ in $T_{0}$ that satisfies $\Pi_{n}$-IND is a $\Pi_{n}$-envelope. The converse also holds for $\Pi_{n+2}^{\mathrm{B}}$-conservative theories. We shall reformulate these facts in the following two results.

Proposition 2.3 Assume that $T \Rightarrow T_{0} \Rightarrow \mathbf{I} \Sigma_{n}+\exp$ and $T$ is a consistent recursively axiomatizable theory. The following conditions are equivalent:

(1) $T$ is $\Pi_{n}$-functional.

(2) There exists a $\Pi_{n}$-envelope of $T$ in $T_{0}$. 
Proof.

$(1) \Rightarrow(2)$ : Since $T \vdash \exp$ and it is recursively axiomatizable, this part follows by [4, Theorem 5.8].

$(2) \Rightarrow(1)$ : By Theorem 1.3 it is enough to prove that $T$ is closed under $\Sigma_{n+1}$-collection rule. Therefore, let $\psi(x, y) \in \Sigma_{n+1}$ such that $T \vdash \forall x \exists y \psi(x, y)$ and $\varphi(u, x, y) \in \Sigma_{n+1}$ a $\Pi_{n}$-envelope of $T$ in $T_{0}$. Then, there is $k \in \omega$ such that

$$
T_{0} \vdash \varphi(k, x, y) \rightarrow(\exists z<y) \psi(x, z) .
$$

Since $T \vdash \forall x \exists y \varphi(k, x, y)$ and $T$ extends $T_{0}$, we have

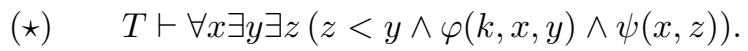

In addition, $T$ also proves that $\varphi(k, x, y)$ defines a nondecreasing function, so, from $(\star)$ it follows that

$$
T \vdash \forall u \exists v(\forall x \leq u)(\exists y \leq v) \psi(x, y),
$$

as required.

Theorem 2.4 Assume that $T \Rightarrow T_{0} \Rightarrow \mathbf{I} \Sigma_{n}+\exp$ and $T$ is a consistent recursively axiomatizable theory. The following conditions are equivalent:

(1) $T$ is $\Pi_{n+2}^{\mathbf{B}}$-conservative.

(2) There exists a (and therefore every) $\Pi_{n}$-envelope of $T$ in $T_{0}$ that satisfies $\Pi_{n}$-IND.

Proof. This result is essentially proved in [4, Theorem 5.5]. We will include the proof here for the sake of completeness.

$(1) \Rightarrow(2)$ : In the proof we use some basic results on recursive saturation and the standard system of a model, $\operatorname{SSy}(\mathfrak{A})$, see [7] for details. By (1), $T$ is $\Pi_{n}$-functional; so, by Proposition 2.3 there exists $\varphi(u, x, y) \in \Sigma_{n+1}$, a $\Pi_{n}$-envelope of $T$ in $T_{0}$. In order to show that $\varphi(u, x, y)$ satisfies property $\Pi_{n}$-IND it is enough to see that IND-(i) $\Rightarrow$ IND-(ii). We follow the proof of [7, Theorem 14.7]. Let $\mathfrak{A} \vDash T_{0}$ countable, nonstandard and let $a, b \in \mathfrak{A}$ such that $\mathfrak{A} \vDash(\exists y<b) \varphi(k, a, y)$, for all $k \in \omega$. Let

$$
T^{\prime}=T+\mathbf{B} \Sigma_{n+1}+\mathbf{c}+\left\{\forall \vec{z} \psi(\mathbf{c}, \vec{z}): \psi(x, \vec{z}) \in \Sigma_{n}, \mathfrak{A} \vDash(\forall \vec{z} \leq b) \psi(a, \vec{z})\right\} .
$$

By (1), as in [7, proof of Theorem 14.7], it follows that $T^{\prime}$ is consistent. Since $\mathfrak{A} \vDash \mathbf{I} \Sigma_{n}+\exp$, the $\Sigma_{n}$-type of $a, b$ in $\mathfrak{A}$ belongs to $\operatorname{SSy}(\mathfrak{A})$; hence,

$$
\left\{\ulcorner\forall \vec{z} \psi(\mathbf{c}, \vec{z})\urcorner: \psi \in \Sigma_{n}, \mathfrak{A} \vDash(\forall \vec{z} \leq b) \psi(a, \vec{z})\right\} \in \operatorname{SSy}(\mathfrak{A}) .
$$

So, as $T$ is recursively axiomatizable, $T^{\prime} \in \operatorname{SSy}(\mathfrak{A})$. Since $\operatorname{SSy}(\mathfrak{A})$ is a Scott set, there exists $\mathfrak{B} \vDash T^{\prime}$ countable and $\operatorname{SSy}(\mathfrak{A})$-saturated (hence, $\mathfrak{B}$ is recursively saturated). Let $c=\mathfrak{B}(\mathbf{c})$. Then, for each $\theta(x, \vec{z}) \in \Pi_{n}$, if $\mathfrak{B} \vDash \exists \vec{z} \theta(c, \vec{z})$, then $\mathfrak{A} \vDash(\exists \vec{z} \leq b) \theta(a, \vec{z})$. So, by Friedman's theorem, there exists $H: \mathfrak{B} \preceq_{n}^{\mathrm{e}} \mathfrak{A}$ such that $H(c)=a$ and $b \notin H(\mathfrak{B})$. Let $I=H(\mathfrak{B})$. Then $I \vDash T, I \prec_{n}^{\mathrm{e}} \mathfrak{A}$ and $a<I<b$.

(2) $\Rightarrow(1)$ : Let $\varphi(u, x, y) \in \Sigma_{n+1}$ be a $\Pi_{n}$-envelope of $T$ in $T_{0}$ that satisfies $\Pi_{n}$-IND. Let $\psi(x, y) \in \Pi_{n}$ such that $T+\mathbf{B} \Sigma_{n+1} \vdash \forall x \exists y \psi(x, y)$. We must prove that $T \vdash \forall x \exists y \psi(x, y)$. Since $T \vdash \forall x \exists y \varphi(k, x, y)$ for all $k \in \omega$ and $T \Rightarrow T_{0}$, it suffices to see that there is $k \in \omega$ such that

$$
T_{0} \vdash \varphi(k, x, y) \rightarrow(\exists z<y) \psi(x, z) .
$$

Towards a contradiction, assume that for all $k \in \omega, T_{0} \forall \varphi(k, x, y) \rightarrow(\exists z<y) \psi(x, z)$. Then, for each $k \in \omega$, there exist $\mathfrak{A}_{k} \vDash T_{0}$, and $a, b \in \mathfrak{A}_{k}$ such that $\mathfrak{A}_{k} \vDash \varphi(k, a, b) \wedge(\forall z<b) \neg \psi(a, z)$. Let $\mathbf{c}$ and d be two new constants and $T^{\prime}$ the theory

$$
T_{0}+(\forall z<\mathbf{d}) \neg \psi(\mathbf{c}, z)+\{(\exists y<\mathbf{d}) \varphi(j, \mathbf{c}, y): j \in \omega\} .
$$

By compactness, $T^{\prime}$ is consistent. Let $\mathfrak{A}^{\prime} \vDash T^{\prime}$ countable, $\mathfrak{A}$ the restriction of $\mathfrak{A}^{\prime}$ to $\mathcal{L}, a=\mathfrak{A}^{\prime}(\mathbf{c})$ and $b=\mathfrak{A}^{\prime}(\mathbf{d})$. Then $\mathfrak{A} \vDash T_{0}$ and, for each $k \in \omega, \mathfrak{A} \vDash(\exists y<b) \varphi(k, a, y)$. By hypothesis, there exists $I \vDash T$ such that $I \prec_{n}^{\mathrm{e}} \mathfrak{A}$ and $a<I<b$. As a consequence, we get $I \vDash T+\mathbf{B} \Sigma_{n+1}$; so, there exists $e \in I$ such that $I \vDash \psi(a, e)$. Hence, $\mathfrak{A} \vDash(\exists z<b) \psi(a, z)$. Contradiction. 
Remark 2.5 The proof of (1) $\Rightarrow$ (2) in Theorem 2.4 yields:

1. Condition IND-(ii) is equivalent to the following one:

IND-(ii)': There exists $I \vDash T$ recursively saturated such that $I \prec_{n}^{\mathrm{e}} \mathfrak{A}$ and $a<I<b$.

This well known fact of the general theory of indicators will be extensively used through this work.

2. If there exists a $\Pi_{n}$-envelope of $T$ in $T_{0}$ that satisfies $\Pi_{n}$-IND, then every $\Pi_{n}$-envelope of $T$ in $T_{0}$ satisfies $\Pi_{n}$-IND.

We close this section with some remarks on a special kind of $\Pi_{n}$-functional classes which are useful in order to get a uniform treatment of $\Pi_{n}$-envelopes. We recall the following definitions from [4].

Let $\Gamma$ be a $\Pi_{n}$-functional class. We say that $\Gamma$ is a strong $\Pi_{n}$-functional class if for every model $\mathfrak{A} \vDash \mathbf{I} \Delta_{0}+\Gamma^{*}$ and $I \subset^{\mathrm{e}} \mathfrak{A}$, it holds that

$$
I \prec_{n}^{\mathrm{e}} \mathfrak{A} \Leftrightarrow \text { for each } \varphi(x, y) \in \Gamma \text { and } a \in I \text { there exists } b \in I \text { such that } \mathfrak{A} \vDash \varphi(a, b) .
$$

That is, if $\Gamma$ is a strong $\Pi_{n}$-functional class and $\mathfrak{A} \vDash \mathbf{I} \Delta_{0}+\Gamma^{*}$, then every initial segment of $\mathfrak{A}$ closed (in $\mathfrak{A}$ ) under the family of functions defined by $\Gamma$ is a $\Sigma_{n}$-elementary substructure of $\mathfrak{A}$ (the converse is obviously true). Notice that the notions of $\Pi_{0}$-functional class and strong $\Pi_{0}$-functional class coincide since each initial substructure of a model of $\mathbf{I} \Delta_{0}$ is $\Delta_{0}$-elementary. However, these notions differ for $n>0$. In [4], we studied properties of strong $\Pi_{n}$-functional classes and proved existence results. In particular, elaborating on a construction by R. Kaye in [6], we showed (see [4, Theorem 5.13]) that for each $n \in \omega$, there exists a $\Pi_{n}$-formula $\mathbb{K}_{n}(x)=y$ such that:

1. $\mathbf{I} \Sigma_{n} \vdash \operatorname{IPF}\left(\mathbb{K}_{n}\right) \wedge \forall x \exists y\left(\mathbb{K}_{n}(x)=y\right)$.

2. $\Gamma_{n}=\left\{\mathbb{K}_{n}(x)=y\right\}$ is a strong $\Pi_{n}$-functional class and $\mathbf{I} \Sigma_{n} \vdash(x+1)^{2} \leq \mathbb{K}_{n}(x)$. So, if $\mathfrak{A} \vDash \mathbf{I} \Sigma_{n}$ and $I$ is an initial segment of $\mathfrak{A}$ closed (in $\mathfrak{A}$ ) under the function defined by $\mathbb{K}_{n}$, then $I \prec_{n}^{\mathrm{e}} \mathfrak{A}$.

Strong $\Pi_{n}$-functional classes provide us with a special kind of $\Pi_{n}$-envelopes. Let $\varphi(u, x, y) \in \Pi_{n}$ be a $\Pi_{n}$-envelope of $T$ in $T_{0}$. We say that $\varphi(u, x, y)$ is a strong $\Pi_{n}$-envelope if $\Gamma_{\varphi}$ is a strong $\Pi_{n}$-functional class. In this definition we restrict ourselves to $\Pi_{n}$-formulas, while our definition of a $\Pi_{n}$-envelope deals with $\Sigma_{n+1}$-formulas. This is not an essential restriction. By a straightforward construction, from any $\Pi_{n}$-envelope we can obtain a new $\Pi_{n}$-envelope given by a $\Pi_{n}$-formula (see [4, Remark 5.9]).

Bearing in mind this fact and the formulas $\mathbb{K}_{n}(x)=y$, it is easy to get strong $\Pi_{n}$-envelopes. Indeed, if $T_{0}$ extends $\mathbf{I} \Sigma_{n}$ and $\varphi(u, x, y) \in \Pi_{n}$ is a $\Pi_{n}$-envelope of $T$ in $T_{0}$, then the following $\Pi_{n}$-formula

$$
\left(u=0 \wedge \mathbb{K}_{n}(x)=y\right) \vee\left(u>0 \wedge\left(\exists y_{1}, y_{2} \leq y\right)\left(\mathbb{K}_{n}(x)=y_{1} \wedge \varphi\left(u, x, y_{2}\right) \wedge y=y_{1}+y_{2}\right)\right)
$$

is a strong $\Pi_{n}$-envelope of $T$ in $T_{0}$. So, in what follows we can assume without loss of generality that the $\Pi_{n}$-envelopes involved are strong or are given by $\Pi_{n}$-formulas.

\section{$2.2 \Pi_{n}$-indicators}

In this section we characterize $\Pi_{n}$-functional theories which are $\Pi_{n+2}^{\mathrm{B}}$-conservative by means of indicators, envelopes and the distribution, in countable and recursively saturated models of $T$, of the initial segments which, in turn, are models of $T$.

Let $\mathfrak{A}$ be a countable $\mathcal{L}$-structure and $Q$ a class of initial segments. In [9] an indicator for $Q$ in $\mathfrak{A}$ is defined as a formula $Y(x, y)=z$ such that

1. $\mathfrak{A} \vDash \forall x, y \exists ! z(Y(x, y)=z)$;

2. for each $a, b \in \mathfrak{A},(\exists I \in Q)\left(I \subset^{\mathrm{e}} \mathfrak{A} \wedge a<I<b\right) \Leftrightarrow Y(a, b)>\omega$.

We shall consider the following notion of indicator.

Definition 2.6 We say that a $\Sigma_{n+1}$-formula $Y(x, y)=z$ is a $\Pi_{n}$-indicator for $T$ in $T_{0}$ if

1. $T_{0} \vdash \forall x, y \exists ! z(Y(x, y)=z)$;

2. for each (countable) $\mathfrak{A} \vDash T_{0}$ and $a, b \in \mathfrak{A},(\exists I \vDash T)\left(I \prec_{n}^{\mathrm{e}} \mathfrak{A} \wedge a<I<b\right) \Leftrightarrow Y(a, b)>\omega$.

In order to get a more elegant theory it is usual to consider a stronger notion of indicator, namely well-behaved indicators (see [9] or [7]). We say that $Y(x, y)=z$ is a well-behaved $\Pi_{n}$-indicator if

$$
T_{0} \vdash \forall x, y(Y(x, y) \leq y) \wedge \forall x, y, x^{\prime}, y^{\prime}\left[x \leq x^{\prime} \leq y^{\prime} \leq y \rightarrow Y\left(x^{\prime}, y^{\prime}\right) \leq Y(x, y)\right] .
$$


It is easy to prove that if $T_{0}$ is a $\Pi_{n}$-functional theory, then there exists a $\Pi_{n}$-indicator for $T$ in $T_{0}$ if and only if there exists a well-behaved one. As a matter of fact, for $\Pi_{n}$-functional theories a general theory of $\Pi_{n}$-indicators can be developed as in $[9,12,14]$. The basic results on indicators can be stated in this context as in those papers.

As noticed in the introduction, given a (well-behaved) indicator $Y(x, y)=z$ it is possible to define an envelope $\varphi_{Y}(u, x, y)$. The following result shows that the converse is also true for $\Pi_{n}$-functional theories: given an envelope $\psi(u, x, y)$, we can get a (well-behaved) indicator $Y_{\psi}(x, y)=z$. The key property that allows us to get $\Pi_{n}$-envelopes from $\Pi_{n}$-indicators is $\Pi_{n+2}^{\mathrm{B}}$-conservativeness. Along the proof we shall describe explicitly the construction of $\varphi_{Y}$ and $Y_{\psi}$.

Theorem 2.7 Let $T$ be $a \Pi_{n}$-functional theory such that $T \vdash$ exp. The following conditions are equivalent:

(1) There exists a $\Pi_{n}$-envelope of $T$ in $T$ satisfying $\Pi_{n}$-IND.

(2) There exists a well-behaved $\Pi_{n}$-indicator, $Y(x, y)=z$, for $T$ in $T$ such that for all $k \in \omega$,

$$
T \vdash \forall x \exists y(Y(x, y)>k) .
$$

Proof.

(2) $\Rightarrow(1)$ : Let $\varphi_{Y}(u, x, y)$ be a $\Sigma_{n+1}$-formula equivalent (in $T$ ) to

$$
y=u+\min \{v \geq x: Y(x, v)>u\} .
$$

By definition, for every $k \in \omega, T \vdash \varphi_{Y}(k+1, x, y) \rightarrow(\exists z<y) \varphi_{Y}(k, x, z)$. Let us see now that for every $k \in \omega$, $T \vdash \operatorname{IPF}\left(\varphi_{Y}(k, x, y)\right)$. Indeed, uniqueness follows from the definition of $\varphi_{Y}(u, x, y)$, and in order to prove (in $T$ ) that $\varphi_{Y}(k, x, y)$ defines a nondecreasing function it is enough to observe that if $x_{1} \leq x_{2}$ and $\varphi_{Y}\left(k, x_{2}, y_{2}\right)$, then $Y\left(x_{2}, y_{2}-k\right)>k$ and, since $Y(x, y)=z$ is a well-behaved $\Pi_{n}$-indicator,

$$
k<Y\left(x_{2}, y_{2}-k\right) \leq Y\left(x_{1}, y_{2}-k\right) .
$$

So, if $\varphi_{Y}\left(k, x_{1}, y_{1}\right)$, then $y_{1}-k \leq y_{2}-k$; hence, $y_{1} \leq y_{2}$.

Let $k \in \omega$. Then $Y(x, y)>k$ is a $\Delta_{n+1}(T)$-formula. Since $T$ is a $\Pi_{n}$-functional theory, $T \Rightarrow \mathbf{L} \Delta_{n+1}(T)$; so, as $T \vdash \forall x \exists y(Y(x, y)>k), T \vdash \forall x \exists y \varphi_{Y}(k, x, y)$. This proves that $\varphi_{Y}(u, x, y)$ is a $\Pi_{n}$-q-envelope of $T$ in $T$.

Next we show that $\varphi_{Y}(u, x, y)$ satisfies $\Pi_{n}$-IND for $T$ and $T$. Let $\mathfrak{A} \vDash T$ and $a, b \in \mathfrak{A}$ such that for every $k \in \omega, \mathfrak{A} \vDash(\exists y<b) \varphi_{Y}(k, a, y)$. By the definition of $\varphi_{Y}(u, x, y)$, for each $k \in \omega$ there is $b_{k} \in \mathfrak{A}$ such that $\mathfrak{A} \vDash b_{k}<b \wedge Y\left(a, b_{k}\right)>k$. Since $Y(x, y)=z$ is a well-behaved $\Pi_{n}$-indicator for $T$ in $T$, for every $k \in \omega$,

$$
\mathfrak{A} \vDash k<Y\left(a, b_{k}\right) \leq Y(a, b) .
$$

Hence, $Y(a, b)>\omega$. So, there exists $I \vDash T$ such that $I \prec_{n}^{\mathrm{e}} \mathfrak{A}$ and $a<I<b$.

$(1) \Rightarrow(2)$ : Let $\varphi(u, x, y) \in \Pi_{n}$ be a $\Pi_{n}$-envelope of $T$ in $T$ that satisfies $\Pi_{n}$-IND. Let $\psi(u, x, y) \in \Pi_{n}$ be the formula

$$
\operatorname{Seq}(y) \wedge \lg (y)=x+1 \wedge(\forall j \leq x) \varphi\left(u, j,(y)_{j}\right) .
$$

That is, $\psi(u, x, y)$ expresses that $y$ is a sequence of length $x+1$ and for each $j \leq x$, its $j$ th component, $(y)_{j}$, satisfies $\varphi\left(u, j,(y)_{j}\right)$. Then $\psi(u, x, y)$ is a $\Pi_{n}$-envelope of $T$ in $T$ satisfying $\Pi_{n}$-IND. Moreover,

$(\bullet) \quad T \vdash x_{1} \leq x_{2} \wedge \psi\left(u, x_{2}, y_{2}\right) \rightarrow\left(\exists y_{1} \leq y_{2}\right) \psi\left(u, x_{1}, y_{1}\right)$.

Let $Y_{\psi}(x, y)=z$ be a $\Sigma_{n+1}$-formula equivalent (in $T$ ) to

$$
[y \leq x \wedge z=0] \vee[x<y \wedge z=\max \{w \leq y:(\forall u<w)(\exists v \leq y) \psi(u, x, v)\}] .
$$

Let us see that $Y_{\psi}(x, y)=z$ is a well-behaved $\Pi_{n}$-indicator for $T$ in $T$.

First of all, observe that, as $T \Rightarrow \mathbf{I} \Sigma_{n}, T \vdash \forall x, y \exists ! z\left(Y_{\psi}(x, y)=z\right)$. Now, let $\mathfrak{A} \vDash T$ countable and $a, b \in \mathfrak{A}$. Then

$$
(\exists I \vDash T)\left(I \prec_{n}^{\mathrm{e}} \mathfrak{A}\right) \wedge a<I<b \Leftrightarrow(\forall k \in \omega)(\mathfrak{A} \vDash(\exists y<b) \psi(k, a, y)) \Leftrightarrow Y_{\psi}(a, b)>\omega .
$$


This proves that $Y_{\psi}(x, y)=z$ is a $\Pi_{n}$-indicator for $T$ in $T$. By the definition of $Y_{\psi}(x, y)=z$, it follows that $T \vdash Y_{\psi}(x, y) \leq y$. Let $a^{\prime}, b^{\prime}, c^{\prime} \in \mathfrak{A}$ such that $a \leq a^{\prime}<b^{\prime} \leq b$ and $\mathfrak{A} \vDash Y_{\psi}\left(a^{\prime}, b^{\prime}\right)=c^{\prime}$. Then, by $(\bullet)$,

$$
\mathfrak{A} \vDash\left(\forall u<c^{\prime}\right)\left(\exists y \leq b^{\prime}\right) \psi\left(u, a^{\prime}, y\right) .
$$

Hence, $\mathfrak{A} \vDash Y_{\psi}(a, b) \geq c^{\prime}$. Consequently, $Y_{\psi}(x, y)=z$ is a well-behaved $\Pi_{n}$-indicator. To conclude the proof let us see that:

Claim 2.7.1 For every $k \in \omega, T+\mathbf{B} \Sigma_{n+1} \vdash \forall x \exists y\left(Y_{\psi}(x, y)>k\right)$.

Proof. Let $\mathfrak{A} \vDash T+\mathbf{B} \Sigma_{n+1}$. Without loss of generality we may assume that $\mathfrak{A}$ is countable, recursively saturated and $T \in \operatorname{SSy}(\mathfrak{A})$. Let $\mathbf{c}$ be a new constant and let $T^{\prime}$ be the theory

$$
T+\left\{(\exists \vec{y}<\mathbf{c}) \psi(\vec{y}): \psi(\vec{y}) \in \Pi_{n}, \mathfrak{A} \vDash \exists \vec{y} \psi(\vec{y})\right\} .
$$

By compactness $T^{\prime}$ is consistent. Since $\mathfrak{A}$ is recursively saturated,

$$
\left\{\psi(\vec{y}) \in \Pi_{n}: \mathfrak{A} \vDash \exists \vec{y} \psi(\vec{y})\right\} \in \operatorname{SSy}(\mathfrak{A}) .
$$

Hence, as $T \in \operatorname{SSy}(\mathfrak{A}), T^{\prime} \in \operatorname{SSy}(\mathfrak{A})$. So, there exists $\mathfrak{B} \vDash T^{\prime}$ countable and recursively saturated verifying $\operatorname{SSy}(\mathfrak{A})=\operatorname{SSy}(\mathfrak{B})$. Let $c=\mathfrak{B}(\mathbf{c})$. Then,

$$
\mathfrak{A} \vDash \exists \vec{y} \psi(\vec{y}) \Rightarrow \mathfrak{B} \vDash(\exists \vec{y}<c) \psi(\vec{y}) .
$$

So, by Friedman's immersion theorem, there is $H: \mathfrak{A} \preceq_{n}^{\mathrm{e}} \mathfrak{B}$ such that $c \notin H(\mathfrak{A})$. Therefore, identifying $H(\mathfrak{A})$ and $\mathfrak{A}$, we get $\mathfrak{A} \prec_{n}^{\mathrm{e}} \mathfrak{B}$ and $\mathfrak{B} \vDash T$.

Let $a \in \mathfrak{A}$. Then for each $b \in \mathfrak{B}$ such that $b>\mathfrak{A}$ and $k \in \omega, \mathfrak{B} \vDash Y_{\psi}(a, b)>k$. Then, as $Y_{\psi}(x, y)>k$ is a $\Delta_{n+1}(T)$-formula and $T$ extends $\mathbf{I} \Delta_{n+1}(T)$, by underspill, there exists $d \in \mathfrak{A}$ such that $\mathfrak{B} \vDash Y_{\psi}(a, d)>k$; hence, $\mathfrak{A} \vDash Y_{\psi}(a, d)>k$.

$\square$ (Claim 2.7.1)

By (1) and Theorem 2.4, $T$ is $\Pi_{n+2}^{\mathrm{B}}$-conservative; therefore, by the above claim, we get that for all $k \in \omega$,

$$
T \vdash \forall x \exists y\left(Y_{\psi}(x, y)>k\right),
$$

as required.

By Theorems 2.4 and 2.7, consistent and recursively axiomatizable $\Pi_{n+2}^{\mathrm{B}}$-conservative theories (proving exp) correspond to those theories whose $\Pi_{n}$-envelopes define $\Pi_{n}$-indicators. Moreover, from the above results, we can also derive the following model-theoretic characterization of $\Pi_{n+2}^{\mathrm{B}}$-conservativeness.

Corollary 2.8 Let $T$ be a recursively axiomatizable theory such that $T \vdash \exp$. The following conditions are equivalent:

(1) $T$ is $\Pi_{n+2}^{\mathrm{B}}$-conservative.

(2) For every countable and recursively saturated $\mathfrak{A} \vDash T$ and $a \in \mathfrak{A}$ there is $I \prec_{n}^{\mathrm{e}} \mathfrak{A}$ (proper) such that $I \vDash T$ and $a \in I$.

Proof.

(1) $\Rightarrow(2)$ : Let $\mathfrak{A} \vDash T$ recursively saturated and $a \in \mathfrak{A}$. By Theorems 2.4 and 2.7, there exists a well-behaved $\Pi_{n}$-indicator, $Y(x, y)=z$, for $T$ in $T$ such that for all $k \in \omega$,

$$
T \vdash \forall x \exists y(Y(x, y)>k) .
$$

Clearly, $\mathbf{p}(y)=\{Y(a, y)>k: k \in \omega\}$ is a recursive type over $\mathfrak{A}$. Then, as $\mathfrak{A}$ is recursively saturated, there exists $b \in \mathfrak{A}$ such that $\mathfrak{A} \vDash \mathbf{p}(b)$; that is, for each $k \in \omega, \mathfrak{A} \vDash Y(a, b)>k$. So, $Y(a, b)>\omega$ and, since $Y(x, y)=z$ is a $\Pi_{n}$-indicator, there exists $I \vDash T$ such that $I \prec_{n}^{\mathrm{e}} \mathfrak{A}$ and $a<I<b$.

(2) $\Rightarrow(1)$ : Let $\varphi(x, y) \in \Pi_{n}$ such that $T+\mathbf{B} \Sigma_{n+1} \vdash \forall x \exists y \varphi(x, y)$. Let $\mathfrak{A} \vDash T$ countable and recursively saturated and $a \in \mathfrak{A}$. By (2), there exists $I \vDash T$ such that $a \in I \prec_{n}^{\mathrm{e}} \mathfrak{A}$ and $I \neq \mathfrak{A}$. Then we have $I \vDash T+\mathbf{B} \Sigma_{n+1}$; hence $I \vDash \exists y \varphi(a, y)$. So, $\mathfrak{A} \vDash \exists y \varphi(a, y)$. 


\section{$2.3 \Pi_{n+3}$-axiomatizable theories}

In view of Proposition 2.3 and Theorem 2.4, our problem $(\mathbf{P})$ on the equivalence between $\Pi_{n}$-functional and $\Pi_{n+2}^{\mathrm{B}}$-conservative theories can be reformulated as follows:

$\left(\mathbf{P}^{\prime}\right) \quad$ Assume that $T+\mathbf{B} \Sigma_{n+1}$ is consistent. Are the following conditions equivalent?

(a) There is a $\Pi_{n}$-envelope of $T$ in $T$.

(b) There is a $\Pi_{n}$-envelope of $T$ in $T$ that satisfies $\Pi_{n}$-IND.

Here we obtain a positive answer to $\left(\mathbf{P}^{\prime}\right)$ for recursively $\Pi_{n+3}$-axiomatizable theories (proving exp). As a corollary, we also obtain a model-theoretic proof of Theorem 1.4 for theories proving exp. Our starting point is a positive answer to $\left(\mathbf{P}^{\prime}\right)$ for $\Pi_{n+2}$-axiomatizable theories.

Lemma 2.9 Let $T$ be a $\Pi_{n}$-functional theory and $T_{0}$ an extension of $\mathbf{I} \Sigma_{n}+\exp$. If $T$ is $\Pi_{n+2}$-axiomatizable, then every $\Pi_{n}$-envelope of $T$ in $T_{0}$ satisfies $\Pi_{n}$-IND.

Proof. Let $\varphi(u, x, y) \in \Pi_{n}$ be a $\Pi_{n}$-envelope of $T$ in $T_{0}$. In the proof we will denote the formula $\varphi(u, x, y)$ by $F_{u}(x)=y$.

Let $\mathfrak{A} \vDash T_{0}$ countable and $a, b \in \mathfrak{A}$ such that, for all $k \in \omega, \mathfrak{A} \vDash(\exists y<b)\left(F_{k}(a)=y\right)$. We must prove that there exists $I \vDash T$ such that $I \prec_{n}^{\mathrm{e}} \mathfrak{A}$ and $a<I<b$.

Let $I=\left\{c \in \mathfrak{A}:\right.$ there exists $k \in \omega$ such that $\left.\mathfrak{A} \vDash c<F_{k}(a)\right\}$. Obviously, $a<I<b$. Let us see that $I \vDash T$ and $I \prec_{n} \mathfrak{A}$. First we prove that $I$ is closed (in $\mathfrak{A}$ ) under functions $F_{m}, m \in \omega$; that is

(*) for each $m \in \omega$ and $c \in I$ there exists $d \in I$ such that $\mathfrak{A} \vDash F_{m}(c)=d$.

Now let $c \in I$ and $k \in \omega$ be such that $c<F_{k}(a)$. Since $T \vdash \forall x \exists z\left(\forall x_{0}<F_{k}(x)\right)\left(\exists y_{0}<z\right)\left(F_{m}\left(x_{0}\right)=y_{0}\right)$ and $\left(\forall x_{0}<F_{k}(x)\right)\left(\exists y_{0}<z\right)\left(F_{m}\left(x_{0}\right)=y_{0}\right)$ is a $\Pi_{n}$-formula, there exists $j \in \omega$ such that

$$
T_{0} \vdash F_{j}(x)=y \rightarrow(\exists z<y)\left(\forall x_{0}<F_{k}(x)\right)\left(\exists y_{0}<z\right)\left(F_{m}\left(x_{0}\right)=y_{0}\right) .
$$

Hence, for $x=a$ it holds that $\mathfrak{A} \vDash\left(\exists z<F_{j}(a)\right)\left(\forall x_{0}<F_{k}(a)\right)\left(\exists y_{0}<z\right)\left(F_{m}\left(x_{0}\right)=y_{0}\right)$. So, now for $x_{0}=c$, $\mathfrak{A} \vDash\left(\exists y_{0}<F_{j}(a)\right)\left(F_{m}(c)=y_{0}\right)$, as required.

We show that $I \prec_{n} \mathfrak{A}$. Since $T$ is $\Pi_{n}$-functional, $T$ extends $\mathbf{I} \Sigma_{n}$; so, $T \vdash \forall x \exists y\left(\mathbb{K}_{n}(x)=y\right)$. Hence, there exists $k \in \omega$ such that

$$
T_{0} \vdash F_{k}(x)=y \rightarrow(\exists z<y)\left(\mathbb{K}_{n}(x)=z\right) .
$$

Hence, by $(\star), I$ is closed under the function $\mathbb{K}_{n}$. But, as remarked at the end of Section $2.1,\left\{\mathbb{K}_{n}(x)=y\right\}$ is a strong $\Pi_{n}$-functional class, so $I \prec_{n} \mathfrak{A}$.

Next we show that $I \vDash T$. Let $\psi(x, y) \in \Pi_{n}$ such that $\forall x \exists y \psi(x, y)$ is an axiom of $T$. Then there is $k \in \omega$ such that

$$
T_{0} \vdash F_{k}(x)=y \rightarrow(\exists z<y) \psi(x, z) .
$$

Let $c \in I$. Then $\mathfrak{A} \vDash\left(\exists z<F_{k}(c)\right) \psi(c, z)$. By $(\star), F_{k}(c) \in I$; so, it follows that $I \vDash \exists z \psi(c, z)$ since $I \prec_{n} \mathfrak{A}$. Hence, $I \vDash \forall x \exists y \psi(x, y)$, as required.

This completes the proof of the lemma.

Theorem 2.10 Let $T$ be a $\Pi_{n}$-functional theory and let $T_{0}$ be a $\Pi_{n+2}$-axiomatizable theory such that

$$
T \Rightarrow T_{0} \Rightarrow \mathbf{I} \Sigma_{n}+\mathbf{e x p}
$$

If $T$ is $\Pi_{n+3}$-axiomatizable, then every $\Pi_{n}$-envelope of $T$ in $T_{0}$ satisfies $\Pi_{n}$-IND.

Proof. Let $\varphi(u, x, y) \in \Pi_{n}$ be a strong $\Pi_{n}$-envelope of $T$ in $T_{0}$ and $T^{*}=\operatorname{Th}_{\Pi_{n+2}}(T)$. First of all let us observe that $\varphi(u, x, y)$ is a $\Pi_{n}$-envelope of $T^{*}$ in $T_{0}$ and also, as $T^{*}$ extends $T_{0}$, of $T^{*}$ in $T^{*}$.

Let $\mathfrak{A} \vDash T_{0}$ countable and $a, b \in \mathfrak{A}$ such that for all $k \in \omega, \mathfrak{A} \vDash(\exists y<b) \varphi(k, a, y)$. We must prove that there exists $I \vDash T$ such that $I \prec_{n}^{\mathrm{e}} \mathfrak{A}$ and $a<I<b$. 
Let $\left\{a_{k}: k \in \omega\right\}$ be an enumeration of all the elements of $\mathfrak{A}$ less than $b$ such that $a_{0}=a$, and let

$$
\left\{\theta_{k}(x, y, z) \in \Sigma_{n}: k \in \omega\right\}
$$

be an enumeration of the $\Sigma_{n}$-formulas such that $T \vdash \forall x \exists y \forall z \theta_{k}(x, y, z)$. We shall define a sequence of initial segments $\left\{I_{k} \prec_{n}^{\mathrm{e}} \mathfrak{A}: k \in \omega\right\}$ and two sequences of elements of $\mathfrak{A}$,

$$
\left\{b_{k} \in \mathfrak{A}: a \leq b_{k}<b\right\} \quad \text { and } \quad\left\{d_{k} \in \mathfrak{A}: a \leq d_{k}<b\right\}
$$

such that for each $k \in \omega$,

1. $d_{k+1} \geq d_{k}$ and $a, d_{k} \in \bigcap_{j \in \omega} I_{j}$;

2. $b_{k+1}<I_{k}<b_{k}$;

3. $I_{k+1} \prec_{n}^{\mathrm{e}} I_{k} \prec_{n}^{\mathrm{e}} \mathfrak{A}$;

4. $I_{k} \vDash T^{*}$ is recursively saturated;

5. for each $i \leq k$, if $a_{i} \in I_{k}$, then $I_{k} \vDash\left(\exists y_{0}, \ldots, y_{k} \leq d_{k}\right) \forall z\left(\bigwedge_{j \leq k} \theta_{j}\left(a_{i}, y_{j}, z\right)\right)$.

Properties 1. -5 . will turn evident from the definition. We proceed by recursion on $k \in \omega$.

$k=0$ : Since $\varphi(u, x, y)$ is a $\Pi_{n}$-envelope of $T^{*}$ in $T_{0}$, by Lemma 2.9, there exists $I_{0}^{\prime} \vDash T^{*}$ such that $I_{0}^{\prime} \prec_{n}^{\mathrm{e}} \mathfrak{A}$ and $a<I_{0}^{\prime}<b$. By Remark 2.5 we can assume that $I$ is recursively saturated. We denote the formula $\varphi(u, x, y)$ by $F_{u}(x)=y$. Since $T \vdash \forall x \exists y \forall z \theta_{0}(x, y, z)$, for each $m \in \omega$,

$$
T^{*} \vdash \forall x \exists z(\exists y<z)\left[F_{m}(\max (x, y))<z \wedge\left(\forall z^{\prime}<z\right) \theta_{0}\left(x, y, z^{\prime}\right)\right] .
$$

Let $\psi\left(u, a_{0}, z\right) \in \Sigma_{0}\left(\Sigma_{n}\right)$ be the formula

$$
\begin{aligned}
(\exists y<z)\left(F_{u}\left(\max \left(a_{0}, y\right)\right)<z\right. & \wedge\left(\forall z^{\prime}<z\right) \theta_{0}\left(a_{0}, y, z^{\prime}\right) \\
& \left.\wedge\left(\forall u^{\prime}<u\right)\left[F_{u^{\prime}}\left(\max \left(a_{0}, y\right)\right)<F_{u}\left(\max \left(a_{0}, y\right)\right)\right]\right) .
\end{aligned}
$$

Then $\mathbf{p}_{0}(z)=\left\{\psi\left(m, a_{0}, z\right): m \in \omega\right\}$ is a recursive type over $I_{0}^{\prime}$; hence, as $I_{0}^{\prime}$ is recursively saturated, there exists $b_{0} \in I_{0}^{\prime}$ realizing $\mathbf{p}_{0}(z)$ in $I_{0}^{\prime}$; that is, for all $m \in \omega, I_{0}^{\prime} \vDash \psi\left(m, a_{0}, b_{0}\right)$. Since $\psi$ is $\Sigma_{0}\left(\Sigma_{n}\right)$, by overspill, there exists $q>\omega$ such that $I_{0}^{\prime} \vDash \psi\left(q, a_{0}, b_{0}\right)$. Let $d_{0}<b_{0}$ such that

$$
\begin{aligned}
I_{0}^{\prime} \vDash F_{q}\left(\max \left(a_{0}, d_{0}\right)\right)<b_{0} & \wedge\left(\forall z^{\prime}<b_{0}\right) \theta_{0}\left(a_{0}, d_{0}, z^{\prime}\right) \\
& \wedge\left(\forall u^{\prime}<q\right)\left[F_{u^{\prime}}\left(\max \left(a_{0}, d_{0}\right)\right)<F_{q}\left(\max \left(a_{0}, d_{0}\right)\right)\right] .
\end{aligned}
$$

Then, for each $m \in \omega, I_{0}^{\prime} \vDash\left(\exists y<b_{0}\right)\left[F_{m}\left(\max \left(a_{0}, d_{0}\right)\right)=y\right]$. Therefore, as $\varphi(u, x, y)$ is a $\Pi_{n}$-envelope of $T^{*}$ in $T^{*}$, by Lemma 2.9 and Remark 2.5, there exists $I_{0} \vDash T^{*}$ recursively saturated such that $I_{0} \prec_{n}^{\mathrm{e}} I_{0}^{\prime} \prec_{n}^{\mathrm{e}} \mathfrak{A}$ and $\max \left(a_{0}, d_{0}\right)<I_{0}<b_{0}$. Clearly, $I_{0} \vDash\left(\exists y \leq d_{0}\right) \forall z \theta_{0}\left(a_{0}, y, z\right)$.

$k \rightarrow k+1$ : Assume that $I_{k}, b_{k}$ and $d_{k}$ have been defined. We define $I_{k+1}, b_{k+1}$ and $d_{k+1}$ as follows. Let $c_{0}, \ldots, c_{r}$ be an enumeration of $\left\{a_{j} \in I_{k}: j \leq k+1\right\}$. Since

$$
T \vdash \forall x_{0} \cdots \forall x_{r} \exists y_{0,0} \cdots \exists y_{r, k+1}\left(\bigwedge_{i=0}^{r} \bigwedge_{j=0}^{k+1} \forall z \theta_{j}\left(x_{i}, y_{i, j}, z\right)\right),
$$

for each $m \in \omega, T^{*}$ proves that

$$
\forall \vec{x} \forall v \exists z(\exists \vec{y}<z)\left[F_{m}(\max (\vec{x}, v, \vec{y}))<z \wedge \bigwedge_{i=0}^{r} \bigwedge_{j=0}^{k+1}\left(\forall z^{\prime}<z\right) \theta_{j}\left(x_{i}, y_{i, j}, z^{\prime}\right)\right] .
$$

Let $\psi_{k+1}\left(u, c_{0}, \ldots, c_{r}, d_{k}, z\right) \in \Sigma_{0}\left(\Sigma_{n}\right)$ be the following formula

$$
\begin{aligned}
(\exists \vec{y}<z)\left(F_{u}\left(\max \left(\vec{c}, d_{k}, \vec{y}\right)\right)<z\right. & \wedge \bigwedge_{i=0}^{r} \bigwedge_{j=0}^{k+1}\left(\forall z^{\prime}<z\right) \theta_{j}\left(c_{i}, y_{i, j}, z^{\prime}\right) \\
& \left.\wedge\left(\forall u^{\prime}<u\right)\left[F_{u^{\prime}}\left(\max \left(\vec{c}, d_{k}, \vec{y}\right)\right)<F_{u}\left(\max \left(\vec{c}, d_{k}, \vec{y}\right)\right)\right]\right) .
\end{aligned}
$$

Then $\mathbf{p}_{k+1}(z)=\left\{\psi_{k+1}\left(m, \vec{c}, d_{k}, z\right): m \in \omega\right\}$ is a recursive type over $I_{k}$; hence, as $I_{k}$ is recursively saturated, there exists $b_{k+1} \in I_{k}$ realizing $\mathbf{p}_{k+1}(z)$; that is, for all $m \in \omega, I_{k} \vDash \psi_{k+1}\left(m, \vec{c}, d_{k}, b_{k+1}\right)$. By overspill, as $\psi_{k+1}$ is a $\Sigma_{0}\left(\Sigma_{n}\right)$-formula, there exists $q>\omega$ such that $I_{k} \vDash \psi_{k+1}\left(q, \vec{c}, d_{k}, b_{k+1}\right)$. Let $e_{i, j}<b_{k+1}, i \leq r, j \leq k+1$, such that

$$
\begin{aligned}
I_{k} \vDash F_{q}\left(\max \left(\vec{c}, d_{k}, \vec{e}\right)\right)<b_{k+1} & \wedge \bigwedge_{i=0}^{r} \bigwedge_{j=0}^{k+1}\left(\forall z^{\prime}<b_{k+1}\right) \theta_{j}\left(c_{i}, e_{i, j}, z^{\prime}\right) \\
& \wedge\left(\forall u^{\prime}<q\right)\left[F_{u^{\prime}}\left(\max \left(\vec{c}, d_{k}, \vec{e}\right)\right)<F_{q}\left(\max \left(\vec{c}, d_{k}, \vec{e}\right)\right)\right] .
\end{aligned}
$$


Let $d_{k+1}=\max \left(d_{k}, \vec{e}\right)$. Then $b_{k+1}<I_{k}<b_{k}$ and $I_{k} \vDash\left(\exists y<b_{k+1}\right)\left[F_{m}\left(\max \left(\vec{c}, d_{k+1}\right)\right)=y\right]$, for each $m \in \omega$. Therefore, as $\varphi(u, x, y)$ is a $\Pi_{n}$-envelope of $T^{*}$ in $T^{*}$, by Lemma 2.9 and Remark 2.5, there exists $I_{k+1} \vDash T^{*}$ recursively saturated such that $I_{k+1} \prec_{n}^{\mathrm{e}} I_{k}$ and $\max \left(\vec{c}, d_{k+1}\right)<I_{k+1}<b_{k+1}$. Moreover, property 5. follows from

$$
I_{k+1} \vDash\left(\exists \vec{y} \leq d_{k+1}\right)\left(\bigwedge_{i=0}^{r} \bigwedge_{j=0}^{k+1} \forall z \theta_{j}\left(c_{i}, y_{i, j}, z\right)\right) .
$$

This concludes the definition of $I_{k+1}, b_{k+1}$ and $d_{k+1}$. Let $I=\bigcap_{j \in \omega} I_{j}$. Then $a<I<b$. It holds:

Claim 2.10.1 $I \prec_{n}^{\mathrm{e}} \mathfrak{A}$ and $I \vDash T$.

Proof. First of all let us recall that $\varphi(u, x, y)$ is a strong $\Pi_{n}$-envelope and that each $I_{k}$ is closed in $\mathfrak{A}$ under the functions $F_{m}$ defined in $\mathfrak{A}$ by the formulas $\varphi(m, x, y)$. As a consequence, $I$ is also closed (in $\left.\mathfrak{A}\right)$ under those functions. Since $\Gamma_{\varphi}$ is a strong $\Pi_{n}$-functional class, $I \prec_{n}^{\mathrm{e}} \mathfrak{A}$.

Let us see that $I \vDash T$. Let $\theta(x, y, z) \in \Sigma_{n}$ such that $T \vdash \forall x \exists y \forall z \theta(x, y, z)$ and let $c \in I$. Then there exist $i, m \in \omega$ such that $a_{i}=c$ and $\theta(x, y, z)$ is $\theta_{m}(x, y, z)$. Let $k>m, i$. Then $a_{i}=c \in I_{k}$ and, by 5 .,

$$
I_{k} \vDash\left(\exists y_{0}, \ldots, y_{k} \leq d_{k}\right) \forall z\left(\bigwedge_{j=0}^{k} \theta_{j}\left(a_{i}, y_{j}, z\right)\right) .
$$

In particular, $I_{k} \vDash\left(\exists y \leq d_{k}\right) \forall z \theta_{m}(c, y, z)$. Since $d_{k} \in I$, there is $e \in I$ such that $I_{k} \vDash \forall z \theta_{m}(c, e, z)$. Then we have $I \prec_{n}^{\mathrm{e}} I_{k}$, since $I \prec_{n}^{\mathrm{e}} \mathfrak{A}$ and $I \subset^{\mathrm{e}} I_{k} \prec_{n}^{\mathrm{e}} \mathfrak{A}$; hence, $I \vDash \forall z \theta_{m}(c, e, z)$. It follows that $I \vDash \forall x \exists y \forall z \theta(x, y, z)$; so, $I \vDash T$ as required.

The previous claim concludes the proof of the theorem.

Corollary 2.11 Let $T$ be a $\Pi_{n+3}$-axiomatizable and consistent extension of $\mathbf{I} \Sigma_{n}+$ exp. Then

$$
T \text { is } \Pi_{n} \text {-functional } \Leftrightarrow T \text { is } \Pi_{n+2}^{\mathrm{B}} \text {-conservative. }
$$

Proof. By Theorem 1.3, it easily follows that every $\Pi_{n+2}^{\mathrm{B}}$-conservative theory is $\Pi_{n}$-functional. Let us prove the converse. First of all, observe that, by Proposition 2.3 and Theorems 2.4 and 2.10, the result holds if $T$ is recursively axiomatizable. Now we address the general case.

Let $\psi \in \Pi_{n+2}$ be such that $T+\mathbf{B} \Sigma_{n+1} \vdash \psi$. Then there exists a sentence $\theta \in \Pi_{n+3}$ such that $T \vdash \theta$ and $\mathbf{B} \Sigma_{n+1}+\theta \vdash \psi$. Let $T^{\prime}$ be the theory $\left(\mathbf{I} \Sigma_{n}+\exp +\theta\right)+\Sigma_{n+1}-\mathrm{CR}$. Then $T^{\prime}$ is recursively $\Pi_{n+3}$-axiomatizable, proves exp and, as it is closed under $\Sigma_{n+1}$-collection rule, is $\Pi_{n}$-functional. So, as noticed above, by Proposition 2.3 and Theorems 2.4 and 2.10, $T^{\prime}$ is $\Pi_{n+2}^{\mathbf{B}}$-conservative; hence, as $T^{\prime}+\mathbf{B} \Sigma_{n+1} \vdash \psi$, it holds that $T^{\prime} \vdash \psi$. Since $T$ extends $T^{\prime}$, we get that $T \vdash \psi$, as required.

Corollary 2.12 Let $T$ be a recursively $\Pi_{n+3}$-axiomatizable and $\Pi_{n}$-functional theory such that $T \vdash \exp$ and $\mathfrak{A} \vDash T$ is countable and recursively saturated. Then for every $a \in \mathfrak{A}$ there exists $I \prec_{n}^{\mathrm{e}} \mathfrak{A}$ (proper) such that $I \vDash T$ and $a \in I$.

\section{Collection rule vs. collection axioms}

Corollary 2.11 gives Theorem 1.4 for $n \geq 1$. Nevertheless, known existence results for $\Pi_{0}$-envelopes use that exponential function is total. This fact forced us to develop an alternative proof for $n=0$. However, the method used in this new proof does work for every $n \in \omega$. This is why we present our arguments for every $n \in \omega$ and not only for $n=0$. The main idea is to adapt the proof of Theorem 2.10 giving a direct construction of the proper $\Sigma_{n}$-elementary initial segment required there. To this end, the key tool is the following weak notion of $\Pi_{n}$-envelope.

Definition 3.1 A pseudo- $\Pi_{n}$-envelope of $T$ in $T_{0}$ is a sequence $\Gamma=\left\{\varphi_{k}(x, y): k \in \omega\right\}$ of $\Sigma_{n+1}$-formulas satisfying

1. $T \vdash \Gamma^{*}$ and for all $k \in \omega, T_{0} \vdash \varphi_{k+1}(x, y) \rightarrow(\exists z<y) \varphi_{k}(x, z)$;

2. for each $\psi(x, y) \in \Pi_{n}$ such that $T \vdash \forall x \exists y \psi(x, y)$ there exists $k \in \omega$ such that

$$
T_{0} \vdash \varphi_{k}(x, y) \rightarrow(\exists z<y) \psi(x, z) .
$$

Pseudo- $\Pi_{n}$-envelopes will provide us with a reduction of $\mathbf{B} \Sigma_{n+1}$ to $\Sigma_{n+1}$-collection rule. We shall thus obtain a model-theoretic proof of [2, Theorem 3.2], proved there by a cut-elimination argument. 
Remark 3.2 A pseudo- $\Pi_{n}$-envelope is, essentially, a "non-uniform" $\Pi_{n}$-envelope. So, results on $\Pi_{n}$-envelopes not depending on their uniform character also hold for pseudo- $\Pi_{n}$-envelopes. In particular, if $\Gamma$ is a pseudo- $\Pi_{n}$-envelope of $T$ in $T_{0}$, by the very definition it follows that:

(i) If $T \Rightarrow T_{0}$, then $\operatorname{Th}_{\Pi_{n+2}}(T)=\mathrm{Th}_{\Pi_{n+2}}\left(T_{0}+\Gamma^{*}\right)$.

(ii) If $\Gamma \subseteq \Pi_{n}$ and $T+\mathbf{I} \Sigma_{n}$ is consistent, then $\Gamma$ is a $\Pi_{n}$-functional class.

Definition 3.3 Let $\Gamma=\left\{\varphi_{k}(x, y): k \in \omega\right\}$ be a sequence of $\Sigma_{n+1}$-formulas. We say that $\Gamma$ satisfies $\Pi_{n}$-IND for $T$ and $T_{0}$ in recursively saturated models if for each $\mathfrak{A} \vDash T_{0}$ countable and recursively saturated and $a, b \in \mathfrak{A}$, the following conditions are equivalent:

IND-(i): For all $k \in \omega, \mathfrak{A} \vDash(\exists y<b) \varphi_{k}(a, y)$.

IND-(ii): There exists $I \vDash T$ such that $I \prec_{n}^{\mathrm{e}} \mathfrak{A}$ and $a<I<b$.

Remark 3.4 By the proof of $(2) \Rightarrow$ (1) in Theorem 2.4, if $\Gamma$ is a sequence of $\Sigma_{n+1}$-formulas that verifies condition 1 . in the definition of pseudo- $\Pi_{n}$-envelope and satisfies $\Pi_{n}$-IND in recursively saturated models, then $\Gamma$ is a pseudo- $\Pi_{n}$-envelope and $T$ is $\Pi_{n+2}^{\mathrm{B}}$-conservative.

Remark 3.5 (The class $\Gamma(\theta)$ ) The next construction associates a pseudo- $\Pi_{n}$-envelope to each $\Sigma_{n}$-formula $\theta(x, y, z)$ such that $\forall x \exists y \forall z \theta(x, y, z)$ is provable in a $\Pi_{n}$-functional theory. In order to ensure that initial segments closed under the functions defined by the pseudo- $\Pi_{n}$-envelope are $\Sigma_{n}$-elementary substructures, we shall use the auxiliary functions $\mathbb{K}_{n}(x)=y$ discussed at the end of Section 2.1.

Let $\theta(x, y, z) \in \Sigma_{n}$ such that $\forall x \exists y \forall z \theta(x, y, z)$ is provable in some $\Pi_{n}$-functional theory. Let

$$
\Gamma(\theta)=\left\{\varphi_{k}(x, y): k \in \omega\right\}
$$

where, for each $k \in \omega, \varphi_{k}(x, y)$ is a $\Sigma_{0}\left(\Sigma_{n}\right)$-formula defining the function $G_{k}^{\theta}(x)=y$ given by

$$
\begin{aligned}
G_{0}^{\theta}(x)=(\mu z)\left(( \forall x _ { 0 } \leq x ) ( \exists y _ { 0 } < z ) \left[\mathbb{K}_{n}\left(\max \left(x, y_{0}\right)\right)<z \wedge\right.\right. & \left.\left.\left(\forall z^{\prime}<z\right) \theta\left(x_{0}, y_{0}, z^{\prime}\right)\right]\right), \\
G_{k+1}^{\theta}(x)=(\mu z)\left(\left(\forall x_{0}, \ldots, x_{k+1} \leq x\right)\left(\exists y_{0}, \ldots, y_{k+1}<z\right)\right. & \left(G_{k}^{\theta}\left(\mathbb{K}_{n}(\max (x, \vec{y}))\right)<z\right. \\
& \left.\left.\wedge \bigwedge_{j=0}^{k+1}\left(\forall z^{\prime}<z\right) \theta\left(x_{j}, y_{j}, z^{\prime}\right)\right)\right) .
\end{aligned}
$$

We shall prove that $\Gamma(\theta)$ is a pseudo- $\Pi_{n}$-envelope of $\mathbf{B} \Sigma_{n+1}+\forall x \exists y \forall z \theta(x, y, z)$ in $\mathbf{I} \Sigma_{n}+\Gamma(\theta)^{*}$ and satisfies $\Pi_{n}$-IND in recursively saturated models.

Lemma 3.6 Let $\theta(x, y, z) \in \Sigma_{n}, T^{\prime}$ be a $\Pi_{n}$-functional theory such that $T^{\prime} \vdash \forall x \exists y \forall z \theta(x, y, z)$ and $\Gamma(\theta)$ as above. Then we have:

(1) $T^{\prime} \vdash \Gamma(\theta)^{*}$. So, $T^{\prime}$ extends $\mathbf{I} \Sigma_{n}+\Gamma(\theta)^{*}$.

(2) For all $k \in \omega, \mathbf{I} \Sigma_{n}+\Gamma(\theta)^{*} \vdash G_{k+1}^{\theta}(x)=y \rightarrow(\exists z<y)\left(G_{k}^{\theta}(x)=z\right)$.

Pro of. Property (2) easily follows from definition. Let us prove (1). We show by induction on $m$ that for all $m \in \omega, T^{\prime} \vdash \operatorname{IPF}\left(G_{m}^{\theta}(x)=y\right) \wedge \forall x \exists y\left(G_{m}^{\theta}(x)=y\right)$.

$m=0$ : Let $\theta_{0}\left(x, x_{0}, z\right)$ be the $\Sigma_{0}\left(\Sigma_{n}\right)$-formula

$$
\left(\exists y_{0}<z\right)\left[\mathbb{K}_{n}\left(\max \left(x, y_{0}\right)\right)<z \wedge\left(\forall z^{\prime}<z\right) \theta\left(x_{0}, y_{0}, z^{\prime}\right)\right] .
$$

Since $T^{\prime} \vdash \forall x \exists y \forall z \theta(x, y, z), T^{\prime} \vdash \forall x \forall x_{0} \exists z \theta_{0}\left(x, x_{0}, z\right)$ and, as a consequence, $\exists z\left(\theta_{0}\left(x, x_{0}, z\right) \in \Delta_{n+1}\left(T^{\prime}\right)\right)$. Since $T^{\prime}$ extends $\mathbf{B}^{*} \Delta_{n+1}\left(T^{\prime}\right)$, it holds that $T^{\prime} \vdash \forall u \exists z\left(\forall x_{0} \leq u\right)\left(\exists z_{0} \leq z\right) \theta_{0}\left(x, x_{0}, z_{0}\right)$.

In particular, $T^{\prime} \vdash \forall x \exists z\left(\forall x_{0} \leq x\right)\left(\exists z_{0} \leq z\right) \theta_{0}\left(x, x_{0}, z_{0}\right)$. Since $T^{\prime}$ is an extension of $\mathbf{I} \Sigma_{n}$,

$$
T^{\prime} \vdash \forall x \exists v\left(v=(\mu z)\left(\left(\forall x_{0} \leq x\right)\left(\exists z_{0} \leq z\right) \theta_{0}\left(x, x_{0}, z_{0}\right)\right)\right) .
$$

This proves that $T^{\prime} \vdash \forall x \exists y\left(G_{0}^{\theta}(x)=y\right)$. Uniqueness follows from the definition of $G_{0}^{\theta}$.

Finally, we must prove now that $G_{0}^{\theta}$ is a nondecreasing function. We reason in $T^{\prime}$. Let $x_{0}, x_{1}, x_{2}$ be such that $x_{0} \leq x_{1} \leq x_{2}$. Then $x_{0} \leq x_{2}$; thus, there exists $y<G_{0}^{\theta}\left(x_{2}\right)$ such that

$$
\mathbb{K}_{n}\left(\max \left(x_{2}, y\right)\right)<G_{0}^{\theta}\left(x_{2}\right) \wedge\left(\forall z^{\prime}<G_{0}^{\theta}\left(x_{2}\right)\right) \theta_{0}\left(x_{0}, y, z^{\prime}\right) .
$$

Since $\mathbb{K}_{n}(x)=y$ defines a nondecreasing function, $\mathbb{K}_{n}\left(\max \left(x_{1}, y\right)\right)<G_{0}^{\theta}\left(x_{2}\right) \wedge\left(\forall z^{\prime}<G_{0}^{\theta}\left(x_{2}\right)\right) \theta_{0}\left(x_{0}, y, z^{\prime}\right)$. Hence, $G_{0}^{\theta}\left(x_{1}\right) \leq G_{0}^{\theta}\left(x_{2}\right)$.

$m \rightarrow m+1:$ To prove that $T^{\prime} \vdash \operatorname{IPF}\left(G_{m+1}^{\theta}(x)=y\right) \wedge \forall x \exists y\left(G_{m+1}^{\theta}(x)=y\right)$, we can easily adapt our previous argument for $m=0$. 
Theorem 3.7 Let $\theta(x, y, z) \in \Sigma_{n}$ and $\Gamma(\theta)$ as above. Then we have:

(a) $\mathbf{B} \Sigma_{n+1}+\forall x \exists y \forall z \theta(x, y, z)$ is consistent.

(b) The class $\Gamma(\theta)$ is a pseudo- $\Pi_{n}$-envelope of $\mathbf{B} \Sigma_{n+1}+\forall x \exists y \forall z \theta(x, y, z)$ in $\mathbf{I} \Sigma_{n}+\Gamma(\theta)^{*}$ satisfying $\Pi_{n}$-IND in recursively saturated models.

(c) $\operatorname{Th}_{\Pi_{n+2}}\left(\mathbf{B} \Sigma_{n+1}+\forall x \exists y \forall z \theta(x, y, z)\right)=\operatorname{Th}_{\Pi_{n+2}}\left(\mathbf{I} \Sigma_{n}+\Gamma(\theta)^{*}\right)$.

Pro of. Part (c) follows from the previous ones: By (a), $\mathbf{B} \Sigma_{n+1}+\forall x \exists y \forall z \theta(x, y, z)$ is $\Pi_{n}$-functional; so, by Lemma 3.6, $\mathbf{B} \Sigma_{n+1}+\forall x \exists y \forall z \theta(x, y, z)$ extends $\mathbf{I} \Sigma_{n}+\Gamma(\theta)^{*}$. Hence, (c) follows by part (b) and Remark 3.2(i). Now we prove parts (a) and (b).

Let us denote $\mathbf{B} \Sigma_{n+1}+\forall x \exists y \forall z \theta(x, y, z)$ by $T_{1}$ and $\mathbf{I} \Sigma_{n}+\Gamma(\theta)^{*}$ by $T_{0}$. First we prove that

$(\bullet) \quad \Gamma(\theta)$ satisfies $\Pi_{n}$-IND for $T_{1}$ and $T_{0}$ in recursively saturated models.

Let $\mathfrak{A} \vDash T_{0}$ be countable and recursively saturated and $a, b \in \mathfrak{A}$, such that $\mathfrak{A} \vDash(\exists y<b)\left(G_{k}^{\theta}(a)=y\right)$, for all $k \in \omega$. In order to get $(\bullet)$ we prove that there exists $I \vDash T_{1}$ such that $I \prec_{n}^{\mathrm{e}} \mathfrak{A}$ and $a<I<b$.

Let $\left\{a_{k}: k \in \omega\right\}$ be an enumeration of all the elements of $\mathfrak{A}$ less than $b$ such that $a_{0}=a$. We shall define a sequence of elements $\left\{c_{k}<b: k \in \omega\right\}$ and two sequences $\left\{b_{k} \in \mathfrak{A}: a \leq b_{k}<b\right\}$ and $\left\{d_{k} \in \mathfrak{A}: a \leq d_{k}<b\right\}$ such that for each $k \in \omega$,

1. $d_{k+1} \geq d_{k}$ and $b_{k+1} \leq b_{k}$;

2. $a_{0}=c_{0}, c_{k}<b_{k}$ and, for all $m \in \omega, G_{m}^{\theta}\left(\max \left(c_{0}, \ldots, c_{k}, d_{k}\right)\right)<b_{k}$;

3. $\mathfrak{A} \vDash\left(\exists y_{0}, \ldots, y_{k} \leq d_{k}\right)\left(\forall z<b_{k}\right)\left(\bigwedge_{j=0}^{k} \theta\left(c_{j}, y_{j}, z\right)\right)$.

We proceed by recursion on $k \in \omega$.

$k=0$ : Let $c_{0}=a_{0}$. Observe that for each $m \in \omega$,

$$
T_{0} \vdash \forall x \exists z\left(\forall x_{0} \leq x\right)\left(\exists y_{0}<z\right)\left[G_{m}^{\theta}\left(\mathbb{K}_{n}\left(\max \left(x, y_{0}\right)\right)\right)<z \wedge\left(\forall z^{\prime}<z\right) \theta\left(x_{0}, y_{0}, z^{\prime}\right)\right] .
$$

For each $m \in \omega$, let $\psi\left(m, c_{0}, y_{0}, z, b\right) \in \Sigma_{0}\left(\Sigma_{n}\right)$ be the formula

$$
y_{0}<z<b \wedge G_{m}^{\theta}\left(\mathbb{K}_{n}\left(\max \left(c_{0}, y_{0}\right)\right)\right)<z \wedge\left(\forall z^{\prime}<z\right) \theta\left(c_{0}, y_{0}, z^{\prime}\right) .
$$

Then $\mathbf{p}_{0}\left(y_{0}, z\right)=\left\{\psi\left(m, c_{0}, y_{0}, z, b\right): m \in \omega\right\}$ is a recursive type over $\mathfrak{A}$; hence, as $\mathfrak{A}$ is recursively saturated, there exist $d_{0}, b_{0} \in \mathfrak{A}$ realizing $\mathbf{p}_{0}\left(y_{0}, z\right)$ in $\mathfrak{A}$; that is, for all $m \in \omega, \mathfrak{A} \vDash \psi\left(m, c_{0}, d_{0}, b_{0}, b\right)$. Then, for all $m \in \omega$, $G_{m}^{\theta}\left(\max \left(c_{0}, d_{0}\right)\right)<b_{0}$ and, clearly, $\mathfrak{A} \vDash\left(\exists y_{0} \leq d_{0}\right)\left(\forall z^{\prime}<b_{0}\right) \theta\left(c_{0}, y_{0}, z^{\prime}\right)$.

$k \rightarrow k+1$ : Assume that $c_{k}, b_{k}$ and $d_{k}$ have been defined. We define $c_{k+1}, b_{k+1}$ and $d_{k+1}$ as follows. Let $a^{\prime}=\max \left(\left\{c_{0}, \ldots, c_{k}, d_{k}, a_{k+1}\right\}\right)$. We distinguish two cases:

$\mathrm{C}$ a se 1: There exists $m \in \omega$ such that $\mathfrak{A} \vDash G_{m}^{\theta}\left(a^{\prime}\right) \geq b_{k}$. Then we define $b_{k+1}=b_{k}, c_{k+1}=c_{k}, d_{k+1}=d_{k}$.

Case 2: For all $m \in \omega, \mathfrak{A} \vDash G_{m}^{\theta}\left(a^{\prime}\right)<b_{k}$. Let $c_{k+1}=a_{k+1}$. For each $m \in \omega, T_{0}$ proves that

$$
\forall x \forall v \exists z(\forall \vec{x} \leq x)(\exists \vec{y}<z)\left[G_{m}^{\theta}\left(\mathbb{K}_{n}(\max (x, v, \vec{y}))\right)<z \wedge \bigwedge_{j=0}^{k+1}\left(\forall z^{\prime}<z\right) \theta\left(x_{j}, y_{j}, z^{\prime}\right)\right] .
$$

Let $\psi_{k+1}\left(m, c_{0}, \ldots, c_{k+1}, d_{k}, b_{k}, y_{0}, \ldots, y_{k+1}, z\right) \in \Sigma_{0}\left(\Sigma_{n}\right)$ be the formula

$$
z<b_{k} \wedge y_{0}<z \wedge \cdots \wedge y_{k+1}<z \wedge G_{m}^{\theta}\left(\mathbb{K}_{n}\left(\max \left(\vec{c}, d_{k}, \vec{y}\right)\right)\right)<z \wedge \bigwedge_{j=0}^{k+1}\left(\forall z^{\prime}<z\right) \theta\left(c_{j}, y_{j}, z^{\prime}\right) .
$$

Then $\mathbf{p}_{k+1}(\vec{y}, z)=\left\{\psi_{k+1}\left(m, \vec{c}, d_{k}, b_{k}, \vec{y}, z\right): m \in \omega\right\}$ is a recursive type over $\mathfrak{A}$; hence, as $\mathfrak{A}$ is recursively saturated, there exist $e_{0}, \ldots, e_{k+1}, b_{k+1} \in \mathfrak{A}$ realizing $\mathbf{p}_{k+1}(\vec{y}, z)$ in $\mathfrak{A}$; that is, for all $m \in \omega$,

$$
\mathfrak{A} \vDash \psi_{k+1}\left(m, \vec{c}, d_{k}, b_{k}, \vec{e}, b_{k+1}\right) .
$$

Let $d_{k+1}=\max \left(d_{k}, \vec{e}\right)$.

Then $b_{k+1}<b_{k}$ and for every $m \in \omega, G_{m}^{\theta}\left(\max \left(c_{0}, \ldots, c_{k+1}, d_{k+1}\right)\right)<b_{k+1}$. Moreover, we can check that 3 . holds since we have

$$
\mathfrak{A} \vDash\left(\exists \vec{y} \leq d_{k+1}\right)\left(\bigwedge_{j=0}^{k+1}\left(\forall z<b_{k+1}\right) \theta\left(c_{j}, y_{j}, z\right)\right) .
$$


This concludes the definition of $b_{k+1}, c_{k+1}$ and $d_{k+1}$. Let $I=\left\{c_{k}: k \in \omega\right\}$. It holds that $a<I<b$ and

Claim 3.7.1

(i) $I \prec_{n}^{\mathrm{e}} \mathfrak{A}$. Hence, $I \vDash \mathbf{B} \Sigma_{n+1}$.

(ii) For all $k \in \omega, d_{k} \in I$.

(iii) $I \vDash T_{1}$.

Proof.

(i) First we prove that $I$ is an initial segment of $\mathfrak{A}$. Let $e \in I$ and $c<e$. Then there is $k \in \omega$ such that $c_{k}=e$. Assume that there is no $i<k$ such that $c_{i}=c$. Then, there exists $j \geq k$ such that $a_{j+1}=c$. So,

$$
a_{j+1} \leq \max \left(c_{0}, \ldots, c_{j}, d_{j}\right) .
$$

By 2., for each $m \in \omega$,

$$
G_{m}^{\theta}\left(\max \left(c_{0}, \ldots, c_{j}, d_{j}\right)\right)<b_{j} .
$$

Since $a^{\prime}=\max \left(c_{0}, \ldots, c_{j}, d_{j}\right)=\max \left(c_{0}, \ldots, c_{j}, a_{j+1}, d_{j}\right)$, then by definition $c=a_{j+1}=c_{j+1} \in I$.

Now we prove that $I \prec_{n} \mathfrak{A}$. It is enough to show that for each $k \in \omega, \mathbb{K}_{n}\left(c_{k}\right) \in I$, recall that $(x+1)^{2} \leq \mathbb{K}_{n}(x)$ and $\left\{\mathbb{K}_{n}(x)=y\right\}$ is a strong $\Pi_{n}$-functional class. For each $k, m \in \omega$, it holds that

$$
\mathbb{K}_{n}\left(c_{k}\right) \leq G_{0}^{\theta}\left(c_{k}\right) \leq G_{m}^{\theta}\left(c_{k}\right) \leq G_{m}^{\theta}\left(\max \left(c_{0}, \ldots, c_{k}, d_{k}\right)\right)<b_{k}
$$

Moreover, for all $m, k \in \omega$,

$$
G_{m}^{\theta}\left(\mathbb{K}_{n}\left(c_{k}\right)\right) \leq G_{m+1}^{\theta}\left(c_{k}\right) \leq G_{m+1}^{\theta}\left(\max \left(c_{0}, \ldots, c_{k}, d_{k}\right)\right)<b_{k} .
$$

Hence, we prove that $\mathbb{K}_{n}\left(c_{k}\right) \in I$ as previously: If there is no $i<k$ such that $c_{i}=\mathbb{K}_{n}\left(c_{k}\right)$, then there is $j \geq k$ such that $a_{j+1}=\mathbb{K}_{n}\left(c_{k}\right)$. Since $a^{\prime}=\max \left(c_{0}, \ldots, c_{j}, d_{j}, a_{j+1}\right)=\max \left(c_{0}, \ldots, c_{j}, d_{j}\right)$, then, by definition, $\mathbb{K}_{n}\left(c_{k}\right)=a_{j+1}=c_{j+1} \in I$.

(ii) Since for all $m \in \omega, G_{m}^{\theta}\left(d_{k}\right)<b_{k}$, we have $d_{k} \in I$.

(iii) Let us see that $I \vDash T_{1}$. Since $I \vDash \mathbf{B} \Sigma_{n+1}$, it is enough to prove that $I \vDash \forall x \exists y \forall z \theta(x, y, z)$.

Let $c \in I$. Then there exists $k \in \omega$ such that $c_{k}=c$ and $c_{k}<b_{k}$. By 3 ,,

$$
\mathfrak{A} \vDash\left(\exists y_{0}, \ldots, y_{k} \leq d_{k}\right)\left(\forall z<b_{k}\right)\left(\bigwedge_{j=0}^{k} \theta\left(c_{j}, y_{j}, z\right)\right) .
$$

In particular,

$$
\mathfrak{A} \vDash\left(\exists y \leq d_{k}\right)\left(\forall z<b_{k}\right) \theta(c, y, z) .
$$

Since $d_{k} \in I$ and $I \subset^{\mathrm{e}} \mathfrak{A}$, there exists $e \in I$ such that $\mathfrak{A} \vDash\left(\forall z<b_{k}\right) \theta(c, e, z)$. Since $I \prec_{n}^{\mathrm{e}} \mathfrak{A}$ and $I<b_{k}$, it holds that $I \vDash \forall z \theta(c, e, z)$. This proves that $I \vDash \forall x \exists y \forall z \theta(x, y, z)$, as required.

$\square$ (Claim 3.7.1)

This completes the proof of $(\bullet)$.

Since there exists a $\Pi_{n}$-functional theory (so, consistent) $T^{\prime}$ such that $T^{\prime} \vdash \forall x \exists y \forall z \theta(x, y, z)$, by Lemma 3.6, $T^{\prime}$ extends $T_{0}$; so, $T_{0}$ is consistent. As a consequence, there exists $\mathfrak{A} \vDash T_{0}$ countable and recursively saturated and $a, b \in \mathfrak{A}$, such that $\mathfrak{A} \vDash(\exists y<b)\left(G_{k}^{\theta}(a)=y\right)$, for all $k \in \omega$. Hence, by $(\bullet)$ there exists $I \vDash T_{1}$ and this proves (a).

Finally, part (b) follows from $(\bullet)$ and Remark 3.4.

Proof of The orem 1.4. We only prove (1) $\Rightarrow(2)$. Let $T$ be a $\Pi_{n+3}$-axiomatizable and $\Pi_{n}$-functional theory and $\psi \in \Pi_{n+2}$ a sentence such that $T+\mathbf{B} \Sigma_{n+1} \vdash \psi$. We must prove that $T \vdash \psi$. Let $\theta(x, y, z) \in \Sigma_{n}$ such that $T \vdash \forall x \exists y \forall z \theta(x, y, z)$ and $\mathbf{B} \Sigma_{n+1}+\forall x \exists y \forall z \theta(x, y, z) \vdash \psi$. By Theorem 3.7,

$$
\operatorname{Th}_{\Pi_{n+2}}\left(\mathbf{B} \Sigma_{n+1}+\forall x \exists y \forall z \theta(x, y, z)\right)=\operatorname{Th}_{\Pi_{n+2}}\left(\mathbf{I} \Sigma_{n}+\Gamma(\theta)^{*}\right) ;
$$

so, $\mathbf{I} \Sigma_{n}+\Gamma(\theta)^{*} \vdash \psi$; hence, by Lemma 3.6(1), $T \vdash \psi$.

As a corollary we get [2, Theorem 3.2]:

Corollary 3.8 Let $T$ be a $\Pi_{n+3}$-axiomatized extension of $\mathbf{I} \Delta_{0}$. Then

$$
\operatorname{Th}_{\Pi_{n+2}}\left(T+\mathbf{B} \Sigma_{n+1}\right)=\operatorname{Th}_{\Pi_{n+2}}\left(T+\Sigma_{n+1}-\mathrm{CR}\right) .
$$


Proof. If $T+\Sigma_{n+1}-\mathrm{CR}$ is inconsistent, then there is nothing to prove. So assume that it is consistent and, thus, by Theorem 1.3, $\Pi_{n}$-functional. Let $\psi \in \Pi_{n+2}$ be a sentence such that $T+\mathbf{B} \Sigma_{n+1} \vdash \psi$. Then there exists $\theta(x, y, z) \in \Sigma_{n}$ such that $T \vdash \forall x \exists y \forall z \theta(x, y, z)$ and $\mathbf{B} \Sigma_{n+1}+\forall x \exists y \forall z \theta(x, y, z) \vdash \psi$. Then, by Theorem 3.7, $\mathbf{I} \Sigma_{n}+\Gamma(\theta)^{*} \vdash \psi$. By Lemma 3.6(1), $T^{\prime}=T+\Sigma_{n+1}$-CR is an extension of $\mathbf{I} \Sigma_{n}+\Gamma(\theta)^{*}$. Hence,

$$
T+\Sigma_{n+1}-\mathrm{CR} \vdash \psi,
$$

as required.

In [8], R. Kaye, J. Paris and C. Dimitracopoulos proved that $\mathbf{B} \Sigma_{n+1}$ is a $\Sigma_{n+3}$-conservative extension of its parameter free version, $\mathbf{B} \Sigma_{n+1}^{-}$. A proof-theoretic proof of this result was obtained by L. Beklemishev as a consequence of his analysis of collection rule in [2]. Our work provides a new model-theoretic proof of that conservation result.

Corollary $3.9 \mathbf{B} \Sigma_{n+1}$ is a $\Sigma_{n+3}$-conservative extension of $\mathbf{B} \Sigma_{n+1}^{-}$.

Proof. Let $\psi \in \Sigma_{n+3}$ be a sentence such that $\mathbf{B} \Sigma_{n+1}^{-} \forall \psi$. Then $T^{\prime}=\mathbf{B} \Sigma_{n+1}^{-}+\neg \psi$ is a $\Pi_{n}$-functional theory and $\neg \psi$ is a $\Pi_{n+3}$-sentence provable in $T^{\prime}$. Let $\theta(x, y, z) \in \Sigma_{n}$ such that $\neg \psi$ is $\forall x \exists y \forall z \theta(x, y, z)$. By Theorem 3.7(a), $\mathbf{B} \Sigma_{n+1}+\neg \psi$ is consistent. Hence, $\mathbf{B} \Sigma_{n+1} \nvdash \psi$.

Remark 3.10 Theorem 1.4 is optimal with respect to the syntactical complexity of the axioms of the theory $T$. Below we give examples of $\Sigma_{n+3}$-axiomatizable theories which are $\Pi_{n}$-functional, but not $\Pi_{n+2}^{\mathrm{B}}$-conservative.

1. Let $\varphi \in \Pi_{n+3}$ be a sentence such that $\mathbf{B} \Sigma_{n+1} \vdash \varphi$ and $\mathbf{B} \Sigma_{n+1}^{-} \nvdash \varphi$. Let $T=\mathbf{B} \Sigma_{n+1}^{-}+\neg \varphi$. Since $T$ is a consistent extension of $\mathbf{B} \Sigma_{n+1}^{-}$, then it is $\Pi_{n}$-functional. It is obvious that $T+\mathbf{B} \Sigma_{n+1}$ is inconsistent. So, $T$ is not $\Pi_{n+2}^{\mathrm{B}}$-conservative.

2. Now we give an example where $T+\mathbf{B} \Sigma_{n+1}$ is consistent. Let $\psi \in \Pi_{1}$ such that $\mathbf{I} \Sigma_{n}+\exp \forall \psi$ and $\mathbf{B} \Sigma_{n+1}+\exp +\psi$ is consistent. Let $T$ be the theory

$$
\mathbf{I} \Sigma_{n}+\exp +\left\{\Theta_{B} \rightarrow \psi\right\}
$$

where $\Theta_{B}$ is a $\Pi_{n+3}$-sentence axiomatizing $\mathbf{B} \Sigma_{n+1}+\exp$. It is obvious that $T+\mathbf{B} \Sigma_{n+1}$ is consistent and $T$ is $\Sigma_{n+3}$-axiomatizable. It also holds that

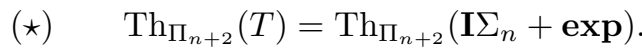

To prove $(\star)$, it is enough to see that $\mathbf{I} \Sigma_{n}+\exp \Rightarrow \mathrm{Th}_{\Pi_{n+2}}(T)$. By way of contradiction, suppose that there is a sentence $\varphi \in \Pi_{n+2}$ such that $T \vdash \varphi$, but $\mathbf{I} \Sigma_{n}+\exp \nvdash \varphi$. We may assume that $\varphi$ is $\forall x \varphi_{0}(x)$, with $\varphi_{0} \in \Sigma_{n+1}$. Then there exists $\mathfrak{A} \vDash \mathbf{I} \Sigma_{n}+\exp +\neg \varphi$ and $p \in \mathfrak{A}$ such that $\mathfrak{A} \vDash \neg \varphi_{0}(p)$. Let us denote by $\mathcal{K}_{n+1}(\mathfrak{A}, p)$ the substructure of $\mathfrak{A}$ determined by the $\Sigma_{n+1}$-definable elements of $\mathfrak{A}$. It is well known that $\mathcal{K}_{n+1}(\mathfrak{A}, p) \prec_{n} \mathfrak{A}$ and $\mathcal{K}_{n+1}(\mathfrak{A}, p) \not \forall \mathbf{B} \Sigma_{n+1}$. So,

$$
\mathcal{K}_{n+1}(\mathfrak{A}, p) \vDash \mathbf{I} \Sigma_{n}+\exp +\neg \varphi .
$$

Since $\mathcal{K}_{n+1}(\mathfrak{A}, p) \not \models \mathbf{B} \Sigma_{n+1}$, we get $\mathcal{K}_{n+1}(\mathfrak{A}, p) \vDash T+\neg \varphi$ and this contradicts $T \vdash \varphi$.

Since $\mathbf{I} \Sigma_{n}+\exp$ is $\Pi_{n}$-functional, by $(\star)$, so is $T$. However, $T$ is not $\Pi_{n+2}^{\mathbf{B}}$-conservative since $T+\mathbf{B} \Sigma_{n+1} \vdash \psi$ and, by $(\star), T \nvdash \psi$.

\section{On $\Sigma_{n+1}$-induction rule}

The analysis of collection rule we have presented in the previous section can be applied with minor changes to $\Sigma_{n+1}$-induction rule. Now the basic result that plays the role of Friedman-Paris' theorem is Parsons' conservation theorem. Actually, here we shall obtain a general version of Parsons' theorem, namely

Theorem 4.1 Let $T$ be a $\Pi_{n+3}$-axiomatizable extension of $\mathbf{I} \Delta_{0}$. Then we have:

$T$ is closed under $\Sigma_{n+1}$-induction rule $\Leftrightarrow T$ is $\Pi_{n+2}^{\mathbf{I}}$-conservative. 
We will derive Theorem 4.1 along the lines used in Section 3 to prove Theorem 1.4. Only two additional facts are needed:

1. If $T$ is closed under $\Sigma_{n+1}$-induction rule, then the class of $\Sigma_{n+1}$-definable functions which are provably total in $T$ is closed under iteration. That is: Let $F(x)=y$ denote a $\Sigma_{n+1}$-formula such that $T \vdash \forall x \exists ! y(F(x)=y)$. Then there exists a $\Sigma_{n+1}$-formula $F^{\prime}(x, z)=y$ such that $T$ proves the formulas $\forall x \forall z \exists ! y\left(F^{\prime}(x, z)=y\right)$ and

$$
\forall x \forall z\left(F^{\prime}(x, 0)=x \wedge F^{\prime}(x, z+1)=F\left(F^{\prime}(x, z)\right)\right) .
$$

The formula $F^{\prime}(x, z)=y$ will be denoted by $F^{z}(x)=y$. Since $T$ is closed under $\Sigma_{n+1}$-induction rule, $T \vdash \exp$ and the usual definition of $F^{z}(x)=y$ works. Moreover, if $T \vdash \operatorname{IPF}(F(x)=y)$, then $T$ proves that the formula $F^{x+1}(x)=y$ also defines a nondecreasing total function.

2. By [2, Lemma 5.1], every theory closed under $\Sigma_{n+1}$-IR is also closed under $\Sigma_{n+1}$-CR; thus, by Theorem 1.3, if $T$ is consistent and closed under $\Sigma_{n+1}$-IR, then $T$ is $\Pi_{n}$-functional.

Bearing in mind these facts, our arguments in Section 3 can be adapted to prove Theorem 4.1. First we associate a pseudo- $\Pi_{n}$-envelope to each $\Sigma_{n}$-formula, $\theta(x, y, z)$, such that $\forall x \exists y \forall z \theta(x, y, z)$ is provable in some consistent theory closed under $\Sigma_{n+1}$-induction rule. For each $k \in \omega, \varphi_{k}(x, y)$ is a $\Sigma_{n+1}$-formula defining the function $H_{\theta, k}(x)=y$ given by

$$
\begin{gathered}
H_{\theta, 0}(x)=(\mu z)\left(\left(\forall x_{0} \leq x\right)\left(\exists y_{0}<z\right)\left(\mathbb{K}_{n}\left(\max \left(x, y_{0}\right)\right)<z \wedge\left(\forall z^{\prime}<z\right) \theta\left(x_{0}, y_{0}, z^{\prime}\right)\right)\right), \\
H_{\theta, k+1}(x)=(\mu z)\left(( \forall \vec { x } \leq x ) ( \exists \vec { y } < z ) \left(H_{\theta, k}^{x+2}\left(\mathbb{K}_{n}(\max (x, \vec{y}))\right)<z\right.\right. \\
\left.\left.\wedge \bigwedge_{j=0}^{k+1}\left(\forall z^{\prime}<z\right) \theta\left(x_{j}, y_{j}, z^{\prime}\right)\right)\right) .
\end{gathered}
$$

We shall prove that $\Gamma_{I}(\theta)=\left\{\varphi_{k}(x, y): k \in \omega\right\}$ is a pseudo- $\Pi_{n}$-envelope of $\mathbf{I} \Sigma_{n+1}+\forall x \exists y \forall z \theta(x, y, z)$ in $\mathbf{I} \Sigma_{n}+\Gamma_{I}(\theta)^{*}$ and satisfies $\Pi_{n}$-IND in recursively saturated models.

Lemma 4.2 Let $\theta(x, y, z) \in \Sigma_{n}$ and let $T^{\prime}$ be a consistent extension of $\mathbf{I} \Delta_{0}$ closed under $\Sigma_{n+1}$-induction rule such that $T^{\prime} \vdash \forall x \exists y \forall z \theta(x, y, z)$ and $\Gamma_{I}(\theta)$ as above. Then we have:

(1) $T^{\prime} \vdash \Gamma_{I}(\theta)^{*}$. So, $T^{\prime}$ extends $\mathbf{I} \Sigma_{n}+\Gamma_{I}(\theta)^{*}$.

(2) For all $k \in \omega, \mathbf{I} \Sigma_{n}+\Gamma_{I}(\theta)^{*} \vdash H_{\theta, k+1}(x)=y \rightarrow(\exists z<y)\left(H_{\theta, k}^{x+2}(x)=z\right)$.

Proof.

(1) We show by induction on $m$ that for all $m \in \omega$,

$$
T^{\prime} \vdash \operatorname{IPF}\left(H_{\theta, m}(x)=y\right) \wedge \forall x \exists y\left(H_{\theta, m}(x)=y\right) .
$$

$m=0$ : Notice that $H_{\theta, 0}=G_{0}^{\theta}$ and by Theorem 1.3, $T^{\prime}$ is $\Pi_{n}$-functional since it is consistent and closed under $\Sigma_{n+1}$-collection rule. So, the result follows by Lemma 3.6.

$m \rightarrow m+1$ : Recall that $T^{\prime}$ is closed under $\Sigma_{n+1}$-IR; so, $H_{\theta, k}^{z}(x)=y$ defines a total function in $T^{\prime}$. Now, we can proceed as in Lemma 3.6.

(2) By (1), $H_{\theta, m}$ is a nondecreasing function; so, part (2) follows from definition.

Theorem 4.3 Let $\theta(x, y, z) \in \Sigma_{n}$ and $\Gamma_{I}(\theta)$ as above. Then we have:

(a) $\mathbf{I} \Sigma_{n+1}+\forall x \exists y \forall z \theta(x, y, z)$ is consistent.

(b) The class $\Gamma_{I}(\theta)$ is a pseudo- $\Pi_{n}$-envelope of $\mathbf{I} \Sigma_{n+1}+\forall x \exists y \forall z \theta(x, y, z)$ in $\mathbf{I} \Sigma_{n}+\Gamma_{I}(\theta)^{*}$ satisfying $\Pi_{n}$-IND in recursively saturated models.

(c) $\operatorname{Th}_{\Pi_{n+2}}\left(\mathbf{I} \Sigma_{n+1}+\forall x \exists y \forall z \theta(x, y, z)\right)=\operatorname{Th}_{\Pi_{n+2}}\left(\mathbf{I} \Sigma_{n}+\Gamma_{I}(\theta)^{*}\right)$.

Proof. We follow the proof of Theorem 3.7. Part (c) follows from parts (a) and (b) as there. Let us denote $\mathbf{I} \Sigma_{n+1}+\forall x \exists y \forall z \theta(x, y, z)$ by $T_{1}$ and $\mathbf{I} \Sigma_{n}+\Gamma_{I}(\theta)^{*}$ by $T_{0}$. Now, we prove that

$(\bullet) \quad \Gamma_{I}(\theta)$ satisfies $\Pi_{n}$-IND for $T_{1}$ and $T_{0}$ in recursively saturated models.

Parts (a) and (b) follow from $(\bullet)$ as in Theorem 3.7.

Let $\mathfrak{A} \vDash T_{0}$ countable and recursively saturated and $a, b \in \mathfrak{A}$, such that $\mathfrak{A} \vDash(\exists y<b)\left(H_{\theta, k}(a)=y\right)$, for all $k \in \omega$. In order to get $(\bullet)$ we show that there exists $I \vDash T_{1}$ such that $I \prec_{n}^{\mathrm{e}} \mathfrak{A}$ and $a<I<b$. 
Let $\left\{\beta_{k}(x, y, v) \in \Pi_{n}: k \in \omega\right\}$ be an enumeration of all $\Pi_{n}$-formulas with exactly those free variables. Let $\left\{\left(a_{k}, r_{k}, s_{k}\right): k \in \omega\right\}$ be an enumeration of all triples of elements of $\mathfrak{A}$ such that $a_{0}=a$ and $a_{k}, r_{k}, s_{k}<b$. We can assume that for each $c<b$ the set $\left\{\beta_{j}\left(x, r_{j}, s_{j}\right): c=a_{j}\right\}$ is the set of all $\Pi_{n}$-formulas with only one free variable and two parameters $<b$. Moreover, we can assume that for all $m \in \omega$,

$$
\mathfrak{A} \vDash(\exists y<b)\left(H_{\theta, m}\left(\max \left(a_{0}, r_{0}, s_{0}\right)\right)=y\right) .
$$

We shall define a sequence of elements $\left\{c_{k}<b: k \in \omega\right\}$ and four sequences $\left\{b_{k} \in \mathfrak{A}: a \leq b_{k}<b\right\}$, $\left\{d_{k} \in \mathfrak{A}: a \leq d_{k}<b\right\},\left\{t_{k} \in \mathfrak{A}: t_{k}<b\right\}$ and $\left\{p_{k} \in \mathfrak{A}: p_{k}<b\right\}$ such that for each $k \in \omega$,

1. $d_{k+1} \geq d_{k}$ and $b_{k+1} \leq b_{k}$;

2. $a_{0}=c_{0}, c_{k}<b_{k}$ and, for all $m \in \omega, H_{\theta, m}\left(\max \left(c_{0}, \ldots, c_{k}, d_{k}\right)\right)<b_{k}$;

3. $\mathfrak{A} \vDash\left(\exists \vec{y} \leq d_{k}\right)\left(\bigwedge_{j=0}^{k}\left(\forall z<b_{k}\right) \theta\left(c_{j}, y_{j}, z\right)\right)$;

4. $\mathfrak{A} \vDash \beta_{k}\left(0, t_{k}, p_{k}\right) \wedge\left(\forall u<c_{k}\right)\left(\left(\exists w<b_{k}\right) \beta_{k}\left(u, w, p_{k}\right) \rightarrow\left(\exists w<b_{k}\right) \beta_{k}\left(u+1, w, p_{k}\right)\right)$ $\rightarrow\left(\forall u \leq c_{k}\right)\left(\exists w \leq d_{k}\right) \beta_{k}\left(u, w, p_{k}\right)$.

We proceed by recursion on $k \in \omega$. We assume that, for all $m \in \omega, H_{\theta, m}\left(\max \left(a_{0}, r_{0}, s_{0}\right)\right)<b$.

$k=0$ : Let $a^{\prime}=\max \left(a_{0}, r_{0}, s_{0}\right)$ and $c_{0}=a_{0}, t_{0}=r_{0}$ and $p_{0}=s_{0}$. Observe that, for each $m \in \omega$,

$$
T_{0} \vdash \forall x \exists z\left(\forall x_{0} \leq x\right)\left(\exists y_{0}<z\right)\left(H_{\theta, m}\left(\mathbb{K}_{n}\left(\max \left(x, y_{0}\right)\right)\right)<z \wedge\left(\forall z^{\prime}<z\right) \theta\left(x_{0}, y_{0}, z^{\prime}\right)\right) .
$$

For each $m \in \omega$, let $\psi_{0}\left(m, y_{0}, z\right)$ be the formula $H_{\theta, m}\left(\mathbb{K}_{n}\left(\max \left(a^{\prime}, y_{0}\right)\right)\right)<z<b \wedge\left(\forall z^{\prime}<z\right) \theta\left(x_{0}, y_{0}, z^{\prime}\right)$.

Then $\mathbf{p}_{0}\left(y_{0}, z\right)=\left\{\psi_{0}\left(m, y_{0}, z\right): m \in \omega\right\}$ is a recursive type over $\mathfrak{A}$; hence, as $\mathfrak{A}$ is recursively saturated, there exist $d_{0}^{\prime}, b_{0}^{\prime} \in \mathfrak{A}$ realizing $\mathbf{p}_{0}\left(y_{0}, z\right)$ in $\mathfrak{A}$; that is, for all $m \in \omega, \mathfrak{A} \vDash \psi\left(m, d_{0}^{\prime}, b_{0}^{\prime}\right)$. Then, for all $m \in \omega$, $H_{\theta, m}\left(\max \left(a^{\prime}, d_{0}^{\prime}\right)\right)<b_{0}^{\prime}$ and, clearly, $\mathfrak{A} \vDash\left(\exists y_{0} \leq d_{0}^{\prime}\right)\left(\forall z^{\prime}<b_{0}^{\prime}\right) \theta\left(c_{0}, y_{0}, z^{\prime}\right)$.

Now, there are three cases. Properties 1. -3 . will follow from definitions. We pay attention to 4.

Case A: $\mathfrak{A} \vDash \neg \beta_{0}\left(0, t_{0}, p_{0}\right)$. Then, we set $d_{0}=d_{0}^{\prime}$ and $b_{0}=b_{0}^{\prime}$. Obviously, 4 . holds.

Cas e B: $\mathfrak{A} \vDash \beta_{0}\left(0, t_{0}, p_{0}\right)$ and there exists $j \in \omega$ such that

$$
\mathfrak{A} \vDash\left(\forall u<c_{0}\right)\left(\left(\exists w_{1}<b_{0}^{\prime}\right) \beta_{0}\left(u, w_{1}, p_{0}\right) \rightarrow\left(\exists w_{2}<H_{\theta, j}\left(\max \left(a^{\prime}, d_{0}^{\prime}, w_{1}\right)\right)\right) \beta_{0}\left(u+1, w_{2}, p_{0}\right)\right) .
$$

Let $d=\max \left(a^{\prime}, d_{0}^{\prime}\right)$. Observe that $T_{0}$ is $\Pi_{n}$-functional; hence, $T_{0}$ extends $\mathbf{I} \Delta_{n+1}\left(T_{0}\right)$. On the other hand, the formula $\left(\exists w<H_{\theta, j}^{x+1}(z)\right) \beta_{0}(u, w, v)$ is $\Delta_{n+1}\left(T_{0}\right)$; so, by induction on $u<c_{0}$ we prove that

$(\star \star) \quad \mathfrak{A} \vDash\left(\forall u \leq c_{0}\right)\left(\exists w<H_{\theta, j}^{u+1}(d)\right) \beta_{0}\left(u, w, p_{0}\right)$.

$u=0$ : It is enough to observe that $\mathfrak{A} \vDash \beta_{0}\left(0, t_{0}, p_{0}\right) \wedge t_{0} \leq a^{\prime} \leq H_{\theta, j}^{u+1}(d)$.

$u \rightarrow u+1$ : From the induction hypothesis and $(\star)$ follows that there exists $w<H_{\theta, j}\left(H_{\theta, j}^{u+1}(d)\right)=H_{\theta, j}^{u+2}(d)$ such that $\beta_{0}\left(u+1, w, p_{0}\right)$, as required.

We define $d_{0}=H_{\theta, j}^{c_{0}+1}(d)$ and $b_{0}=b_{0}^{\prime}$. Observe that $c_{0} \leq d_{0}$ and, by Lemma 4.2(2), for all $m \geq j$,

$$
H_{\theta, m}\left(d_{0}\right) \leq H_{\theta, m}\left(H_{\theta, j}^{d+2}(d)\right) \leq H_{\theta, m}\left(H_{\theta, m+1}(d)\right) \leq H_{\theta, m+1}^{2}(d) \leq H_{\theta, m+2}(d)<b_{0}
$$

This yields property 3. Moreover, $H_{\theta, j}$ is nondecreasing and, by $(\star \star), \mathfrak{A} \vDash\left(\forall u \leq c_{0}\right)\left(\exists w \leq d_{0}\right) \beta_{0}\left(u, w, p_{0}\right)$; so, property 4. also holds.

C a s e $\mathrm{C}: \mathfrak{A} \vDash \beta_{0}\left(0, t_{0}, p_{0}\right)$ and for all $j \in \omega,(\star)$ does not hold. Let $\psi_{0}^{\prime}\left(m, u, w_{1}, z\right)$ be the formula

$$
H_{\theta, m}\left(\max \left(c_{0}, d_{0}^{\prime}, w_{1}\right)\right)<z<b_{0}^{\prime} \wedge u<c_{0} \wedge \beta_{0}\left(u, w_{1}, p_{0}\right) \wedge\left(\forall w_{2}<z\right) \neg \beta_{0}\left(u+1, w_{2}, p_{0}\right) .
$$

Then $\mathbf{q}_{0}\left(u, w_{1}, z\right)=\left\{\psi_{0}^{\prime}\left(m, u, w_{1}, z\right): m \in \omega\right\}$ is a recursive type (since each $H_{\theta, m}$ is a non-decreasing function). So, there exist $e, d_{0}^{\prime \prime}, b_{0}^{\prime \prime}$ such that $\mathfrak{A} \vDash \mathbf{q}_{0}\left(e, d_{0}^{\prime \prime}, b_{0}^{\prime \prime}\right)$. We define $b_{0}=b_{0}^{\prime \prime}$ and $d_{0}=\max \left(d_{0}^{\prime}, d_{0}^{\prime \prime}\right)$. Clearly 4. holds since $\mathfrak{A} \vDash\left(\exists u<c_{0}\right)\left(\left(\exists w_{1}<b_{0}\right) \beta_{0}\left(u, w_{1}, p_{0}\right) \wedge\left(\forall w_{2}<b_{k}\right) \neg \beta_{0}\left(u+1, w_{2}, p_{0}\right)\right)$.

$k \rightarrow k+1$ : Assume that $c_{k}, b_{k}, d_{k}, p_{k}$ and $t_{k}$ have been defined. We define $c_{k+1}, b_{k+1}, d_{k+1}, p_{k+1}$ and $t_{k+1}$ as follows. Let $a^{\prime}=\max \left\{c_{0}, \ldots, c_{k}, d_{k}, a_{k+1}, r_{k+1}, s_{k+1}\right\}$. We distinguish two cases:

Case 1: There exists $m \in \omega$ such that $\mathfrak{A} \vDash H_{\theta, m}\left(a^{\prime}\right) \geq b_{k}$. Then define $b_{k+1}=b_{k}, c_{k+1}=c_{k}, d_{k+1}=d_{k}$, $p_{k+1}=p_{k}$ and $t_{k+1}=t_{k}$. 
Case 2: For all $m \in \omega, \mathfrak{A} \vDash H_{\theta, m}\left(a^{\prime}\right)<b_{k}$. Let $c_{k+1}=a_{k+1}, p_{k+1}=s_{k+1}$ and $t_{k+1}=r_{k+1}$. Then for each $m \in \omega, T_{0}$ proves that

$$
\forall x \exists z(\forall \vec{x} \leq x)(\exists \vec{y}<z)\left(H_{\theta, k}\left(\mathbb{K}_{n}(\max (x, \vec{y}))\right)<z \wedge \bigwedge_{j=0}^{k+1}\left(\forall z^{\prime}<z\right) \theta\left(x_{j}, y_{j}, z^{\prime}\right)\right) .
$$

Let $\psi_{k+1}(m, \vec{y}, z)$ be the formula $H_{\theta, m}\left(\mathbb{K}_{n}\left(\max \left(a^{\prime}, \vec{y}\right)\right)\right)<z<b_{k} \wedge \bigwedge_{j=0}^{k+1}\left(\forall z^{\prime}<z\right) \theta\left(c_{j}, y_{j}, z^{\prime}\right)$. Then

$$
\mathbf{p}_{k+1}(\vec{y}, z)=\left\{\psi_{k+1}(m, \vec{y}, z): m \in \omega\right\}
$$

is a recursive type over $\mathfrak{A}$; as a consequence, there exist $e_{0}, \ldots, e_{k+1}, b_{k+1}^{\prime} \in \mathfrak{A}$, such that for all $m \in \omega$, $\mathfrak{A} \vDash \psi_{k+1}\left(m, \vec{e}, d, b_{k+1}\right)$. Let $d_{k+1}^{\prime}=\max \left(d_{k}, \vec{e}\right)$.

Now, we distinguish three cases:

Case A: $\mathfrak{A} \vDash \neg \beta_{k+1}\left(0, t_{k+1}, p_{k+1}\right)$. Then, we set $d_{k+1}=d_{k+1}^{\prime}$ and $b_{k+1}=b_{k+1}^{\prime}$.

Case $\mathrm{B}: \mathfrak{A} \vDash \beta_{k+1}\left(0, t_{k+1}, p_{k+1}\right)$ and there exists $j \in \omega$ such that

$$
\begin{aligned}
\mathfrak{A} \vDash\left(\forall u<c_{k+1}\right) & \left(\left(\exists w_{1}<b_{k+1}^{\prime}\right) \beta_{k+1}\left(u, w_{1}, p_{k+1}\right)\right. \\
& \left.\rightarrow\left(\exists w_{2}<H_{\theta, j}\left(\max \left(a^{\prime}, d_{k+1}^{\prime}, w_{1}\right)\right)\right) \beta_{k+1}\left(u+1, w_{2}, p_{k+1}\right)\right) .
\end{aligned}
$$

Then, we set $d_{k+1}=H_{\theta, j}^{c_{k+1}+1}\left(\max \left(a^{\prime}, d_{k+1}^{\prime}\right)\right)$ and $b_{k+1}=b_{k+1}^{\prime}$. Properties 1 . -4 . follow as for $k=0$.

Cas e C: $\mathfrak{A} \vDash \beta_{k+1}\left(0, t_{k+1}, p_{k+1}\right)$ and Case B fails. Let $\psi_{k+1}^{\prime}\left(m, u, w_{1}, z\right)$ be the formula

$$
\begin{aligned}
H_{\theta, m}\left(\max \left(a^{\prime}, d_{k+1}^{\prime}, w_{1}\right)\right)<z<b_{k+1}^{\prime} & \wedge u<c_{k+1} \wedge \beta_{k+1}\left(u, w_{1}, p_{k+1}\right) \\
& \wedge\left(\forall w_{2}<z\right) \neg \beta_{k+1}\left(u+1, w_{2}, p_{k+1}\right) .
\end{aligned}
$$

As for $k=0, \mathbf{q}_{k+1}\left(u, w_{1}, z\right)=\left\{\psi_{k+1}^{\prime}\left(m, u, w_{1}, z\right): m \in \omega\right\}$ is a recursive type. So, there exist $e, d_{k+1}^{\prime \prime}, b_{k+1}^{\prime \prime}$ such that $\mathfrak{A} \vDash \mathbf{q}_{k+1}\left(e, d_{k+1}^{\prime \prime}, b_{k+1}^{\prime \prime}\right)$. We define $b_{k+1}=b_{k+1}^{\prime \prime}$ and $d_{k+1}=\max \left(d_{k+1}^{\prime}, d_{k+1}^{\prime \prime}\right)$.

This concludes the definition of the five sequences. Let $I=\left\{c_{k}: k \in \omega\right\}$. Obviously $a<I<b$.

\section{Claim 4.3.1}

(i) $I \prec_{n}^{\mathrm{e}} \mathfrak{A}$.

(ii) For all $k \in \omega, d_{k}, t_{k}, p_{k} \in I$.

(iii) $I \vDash T_{1}$.

Proof. (i) and (ii) are proved as in Theorem 3.7.

(iii) We only prove $I \vDash \mathbf{I} \Sigma_{n+1}$ since $I \vDash \forall x \exists y \forall z \theta(x, y, z)$ can be checked as in Theorem 3.7.

Let $\beta(x, y, p)$ be a $\Pi_{n}$-formula with parameter $p \in I$ and $t \in I$ such that

$$
I \vDash \beta(0, t, p) \wedge \forall x(\exists y \beta(x, y, p) \rightarrow \exists y \beta(x+1, y, p)) .
$$

Let $c \in I$. We must prove that $I \vDash \exists y \beta(c, y, p)$. By construction, there exists $k \in \omega$ such that $c_{k}=c, t_{k}=t$, $p_{k}=p$ and $\beta_{k}=\beta$. Since $I \prec_{n}^{\mathrm{e}} \mathfrak{A}$, by $(\mathrm{H})$, at step $k$ of the construction we are in Case B. So, it follows that $\mathfrak{A} \vDash\left(\forall u \leq c_{k}\right)\left(\exists w \leq d_{k}\right) \beta_{k}\left(u, w, p_{k}\right)$. In particular, $\mathfrak{A} \vDash\left(\exists w \leq d_{k}\right) \beta_{k}\left(c_{k}, w, p_{k}\right)$; hence,

$$
I \vDash\left(\exists w \leq d_{k}\right) \beta(c, w, p)
$$

since $I \prec_{n}^{\mathrm{e}} \mathfrak{A}$ and $d_{k} \in I$.

(Claim 4.3.1)

This completes the proof of $(\bullet)$. Parts (a) and (b) follow from $(\bullet)$ as in Theorem 3.7.

Now Theorem 4.1 can be derived from Theorem 4.3 following the proof of Theorem 1.4 in Section 3. Moreover, modifying accordingly the proofs of Corollaries 3.8 and 3.9 we get

\section{Corollary 4.4}

1. $\mathbf{I} \Sigma_{n+1}$ is a $\Sigma_{n+3}$-conservative extension of $\mathbf{I} \Sigma_{n+1}^{-}$.

2. Let $T$ be a $\Pi_{n+3}$-axiomatizable extension of $\mathbf{I} \Delta_{0}$. Then $T+\Sigma_{n+1}$-IR is $\Pi_{n+2}^{\mathbf{I}}$-conservative.

Acknowledgements The present work was partially supported by the Andalusian Government, grant TIC-137. 


\section{References}

[1] L. Beklemishev, Induction rules, reflection principles, and provably total recursive functions. Ann. Pure Applied Logic 85, 193 - 242 (1997).

[2] L. Beklemishev, A proof-theoretic analysis of collection. Archive Math. Logic 37, 275 - 296 (1998).

[3] A. Cordón-Franco, A. Fernández-Margarit, and F. F. Lara-Martín, On the quantifier complexity of $\Delta_{n+1}(T)$-induction. Archive Math. Logic 43, 371 - 398 (2004).

[4] A. Fernández-Margarit and F. F. Lara-Martín, Induction, minimization and collection for $\Delta_{n+1}(T)$-formulas. Archive Math. Logic 43, 505 - 541 (2004).

[5] P. Hájek and P. Pudlák, Metamathematics of First Order Arithmetic. Perspectives in Mathematical Logic (SpringerVerlag, 1993).

[6] R. Kaye, Diophantine and parameter-free induction. Ph. D. thesis, University of Manchester, 1987.

[7] R. Kaye, Models of Peano Arithmetic (Clarendon Press, 1991).

[8] R. Kaye, J. Paris, and C. Dimitracopoulos, On parameter free induction schemas. J. Symbolic Logic 53, 1082 - 1097 (1988).

[9] L. Kirby, Initial segments of models of arithmetic. Ph. D. thesis, University of Manchester, 1977.

[10] L. Kirby, La méthode des indicatrices et le théorèm d'incompletude. In: Modèles de l'arithmétique (K. McAloon, ed.), Astérisque 73, pp. 5 - 18 (Société Mathématique de France, 1980).

[11] L. Kirby, K. McAloon, and R. Murawski, Indicators, recursive saturation and expandability. Fundamenta Mathematicae 114, 127 - 139 (1981)

[12] L. Kirby and J. Paris, Initial segments of models of Peano's axioms. In: Conference on Set Theory and Hierarchy Theory, Bierutowice, 1976, Lecture Notes in Mathematics 619, pp. 211 - 226 (Springer-Verlag, 1977).

[13] K. McAloon, Models of arithmetic and complexity theory. In: Studies in Complexity Theory (R. Book, ed.), pp. 119 - 226 (Pitman, 1986).

[14] J. Paris, Some independence results for Peano arithmetic. J. Symbolic Logic 43, 725 - 731 (1978).

[15] J. Paris, A hierarchy of cuts in models of Arithmetic. In: Lecture Notes in Mathematics 834, pp. 312 - 337 (SpringerVerlag, 1979).

[16] C. Parsons, On n-quantifier induction. J. Symbolic Logic 37 (3), 466 - 482 (1972). 